\title{
Practical considerations when prescribing a long-acting muscarinic antagonist for patients with COPD
}

This article was published in the following Dove Press journal: International Journal of COPD

\author{
Anthony D D'Urzo' \\ Peter Kardos ${ }^{2}$ \\ Russell Wiseman ${ }^{3}$ \\ 'Department of Family and \\ Community Medicine, Faculty of \\ Medicine, University of Toronto, \\ ON, Canada; ${ }^{2}$ Group Practice and \\ Centre for Allergy, Respiratory and \\ Sleep Medicine, Red Cross Maingau \\ Hospital, Frankfurt, Germany; \\ ${ }^{3}$ Suncoast Medical Centre, Coolum \\ Beach, QLD, Australia
}

\begin{abstract}
COPD is characterized by persistent airflow limitation, progressive breathlessness, cough, and sputum production. Long-acting muscarinic antagonists (LAMAs) are one of the recommended first-choice therapeutic options for patients with COPD, and several new agents have been developed in recent years. A literature search identified 14 published randomized, placebo-controlled studies of the efficacy and safety of LAMAs in patients with COPD, with improvements seen in lung function, exacerbations, breathlessness, and health status. A greater weight of evidence currently exists for glycopyrronium (GLY) and tiotropium than for umeclidinium and aclidinium, especially in terms of exacerbation reductions. To date, there have been few head-to-head clinical studies of the different LAMAs. Available data indicate that GLY and aclidinium have similar efficacy to tiotropium in terms of improving lung function, dyspnea, exacerbations, and health status. Overall, evidence demonstrates that currently available LAMAs provide effective and generally well-tolerated therapy for patients with COPD. Delivery devices for the different LAMAs vary, which may affect individual patient's adherence to and preference for treatment. Subtle differences between individual therapeutic options may be important to individual patients and the final treatment choice should involve physician's and patient's experiences and preferences.
\end{abstract}

Keywords: COPD, long-acting muscarinic antagonist, efficacy, safety, inhaler, adherence

\section{Introduction}

COPD is a common, preventable and treatable disease characterized by persistent respiratory symptoms and airflow limitation. ${ }^{1}$ Damage caused by a combination of small airway disease (obstructive bronchiolitis) and parenchymal destruction (emphysema) results in the characteristic symptoms of COPD: progressive breathlessness on exertion and persistent airflow limitation.

Airflow limitation may be improved using bronchodilators, which alter airway smooth muscle tone, thereby reducing airway resistance and static and dynamic hyperinflation. ${ }^{1-3}$ Both classes of long-acting bronchodilator (long-acting muscarinic antagonists [LAMAs; also known as anticholinergics] and long-acting $\beta_{2}$-agonists [LABAs]) improve lung function, symptoms, health status, exercise tolerance, and exacerbations in COPD patients. ${ }^{1}$ LABAs may be more effective than LAMAs at reducing symptoms (although results are conflicting), ${ }^{4,5}$ while LAMAs may be superior to LABAs for exacerbation prevention. ${ }^{6,7}$ The complementary mechanisms of action of LABAs and LAMAs elicit additive effects on lung function and provide rationale for combining the two classes. ${ }^{1}$ In this review, we focus on the available evidence for the efficacy and safety of LAMAs in the management of COPD.
Correspondence: Anthony D D'Urzo Department of Family and Community Medicine, Faculty of Medicine, University of Toronto, 500 University Avenue, 5th Floor, Toronto, ON, M5G IV7, Canada Tel +I 4166529336

Fax +l 4166520218

Email tonydurzo@sympatico.ca 
In 2002, tiotropium $18 \mu \mathrm{g}$ once daily (qd) was the first LAMA to be approved for the maintenance treatment of patients with COPD, ${ }^{8,9}$ followed by glycopyrronium (GLY) $50 \mu \mathrm{g} \mathrm{qd}$, which was approved in Europe in 2012. ${ }^{10}$ More recently, umeclidinium $65 \mu \mathrm{g} \mathrm{qd}^{11}$ and aclidinium $400 \mu \mathrm{g}$ twice daily (bid) were also approved. ${ }^{9}$

The Global initiative for Obstructive Lung Disease (GOLD) 2018 strategy document contains substantial revisions to the committee's recommendations for diagnosis, classification, and treatment of patients with COPD, including their recommendations for LAMA use. ${ }^{1}$ According to GOLD, LAMA monotherapy may be an appropriate treatment for all patients with COPD, irrespective of the GOLD group to which their disease is assigned. Moreover, a LAMA is recommended as part of a dual or triple combination for those patients with persistent or severe breathlessness or persistent exacerbations, ie, GOLD Groups B-D.

Accordingly, LAMAs may be used as first-choice therapy for patients with few symptoms and no exacerbation history (GOLD Group A). ${ }^{1}$ In this patient group, GOLD recommends either a short-acting bronchodilator or long-acting bronchodilator of either class. ${ }^{1}$ Initial treatment with LAMA or LABA monotherapy is recommended for symptomatic patients at low risk of exacerbation (GOLD Group B), escalating to dual bronchodilation if breathlessness persists. GOLD does not recommend one class of bronchodilator over another for Group B patients and states that choice should depend on individual perception of symptom relief. ${ }^{1}$

GOLD also recommends initial treatment with LAMA monotherapy in patients with an exacerbation history but few symptoms (GOLD Group C). ${ }^{1}$ In both POET (Prevention of Exacerbations with Tiotropium) and INVIGORATE (Indacaterol: Providing Opportunity to Re-engage Patients with Life), the LAMA tiotropium demonstrated a greater effect on annual exacerbation rate than a LABA (salmeterol and indacaterol [IND], respectively) in patients with severe COPD. ${ }^{6,7}$ If patients experience further exacerbations on LAMA monotherapy, GOLD recommends escalating treatment to LABA/ LAMA (preferred choice) or inhaled corticosteroid (ICS)/ LABA (alternative choice). ${ }^{1}$ Group C patients are not commonly studied in clinical trials, and there is little validated information regarding the treatment of these patients.

The Spanish Guidelines for COPD (GesEPOC) also suggest that patients who require a long-acting bronchodilator as monotherapy should use a LAMA in the first instance, based on greater reductions in exacerbations with tiotropium compared with LABAs. ${ }^{12-14}$ In symptomatic patients, the GesEPOC guidelines recommend a combination of LABA/LAMA. ${ }^{15}$
LAMAs have a clear role in treatment recommendations. ${ }^{1}$ However, the rapid arrival of new medications for the management of COPD, each with a distinct delivery inhaler and supporting evidence, has created considerable prescriber confusion. Here, we compare clinical evidence for currently available LAMAs to further clarify appropriate treatment selection and advise practicing physicians on the management of patients with COPD.

\section{Methods}

The PubMed database was searched ([aclidinium OR GLY OR tiotropium OR umeclidinium] AND COPD AND placebo AND randomized) to identify English language publications of studies of currently available LAMAs as the active treatment in patients with COPD. Results were filtered to identify primary publications of randomized, double-blind, placebocontrolled studies of $\geq 6$ months' duration using approved doses of the four available LAMAs. Information was retrieved from the corresponding summary of product characteristics (SmPC) and from regulatory documentation available at the European Medicines Agency (EMA). ${ }^{8-11,16,17}$

\section{Results}

The literature search identified 366 publications, of which 14 were primary publications of double-blind, randomized, placebo-controlled studies of $\geq 6$ months duration for the four LAMAs (Table 1). ${ }^{18-31}$ Of the 14 studies, six studies evaluated tiotropium $18 \mu \mathrm{g}$ qd (HandiHaler ${ }^{\circledR}$ ), two studies evaluated tiotropium $5 \mu \mathrm{g}$ qd (Respimat ${ }^{\circledR}$ ), two studies evaluated GLY $50 \mu \mathrm{g} \mathrm{qd}$, one study evaluated umeclidinium $62.5 \mu \mathrm{g} \mathrm{qd}$, and three studies evaluated aclidinium $400 \mu \mathrm{g}$ bid. Aclidinium has also been evaluated in a 52-week extension to a 12-week randomized, double-blind, placebo-controlled trial (during the extension, patients received aclidinium 200 or $400 \mu \mathrm{g} \mathrm{qd}$, with no placebo arm). ${ }^{32}$ In the USA, GLY $12.5 \mu \mathrm{g}$ has been developed for twice-daily use; ${ }^{33,34}$ however, this is outside the scope of this review.

The outcomes below were included in the majority of studies and provided a basis for comparing efficacy between LAMAs.

\section{Lung function}

Forced expiratory volume in 1 second $\left(\mathrm{FEV}_{1}\right)$ is a validated and important measure of lung function in COPD. Not only is $\mathrm{FEV}_{1}$ a predictor of all-cause mortality ${ }^{1,35}$ but also is correlated to COPD symptoms, exacerbations, and overall health care resource use and costs. ${ }^{36}$

Trough $\mathrm{FEV}_{1}$ (measured 12 hours after the preceding dose of a twice-daily treatment or 24 hours after a once-daily 
Table I Double-blind, randomized, placebo-controlled studies of LAMA therapy with durations of at least 6 months

\begin{tabular}{|c|c|c|c|c|c|}
\hline Study & Duration & $\mathbf{N}$ & $\begin{array}{l}\text { Baseline FEV } \\
\% \text { predicted }^{a}\end{array}$ & Treatments & $\begin{array}{l}\text { Concomitant maintenance } \\
\text { treatment for COPD }\end{array}$ \\
\hline \multicolumn{6}{|c|}{ Tiotropium (via HandiHaler ${ }^{\circledR}$ device) } \\
\hline $\begin{array}{l}\text { Casaburi et al } \\
(2002)^{18}\end{array}$ & I year $\times 2^{b}$ & 921 & $39^{c}$ & $\begin{array}{l}\text { Tiotropium I8 } \mu \mathrm{g} \text { qd } \\
\text { Placebo }(3: 2)\end{array}$ & Theophylline; ICS/OCS \\
\hline $\begin{array}{l}\text { Donohue et al } \\
(2002)^{19}\end{array}$ & 6 months & 623 & $40^{c}$ & $\begin{array}{l}\text { Tiotropium } 18 \mu \mathrm{g} \text { qd } \\
\text { Salmeterol } 50 \mu \mathrm{g} \text { bid } \\
\text { Placebo (I:I:I) }\end{array}$ & ICS/OCS \\
\hline $\begin{array}{l}\text { Niewoehner } \\
\text { et al }(2005)^{20}\end{array}$ & 6 months & 1,829 & $\begin{array}{l}36(\leq 60 \\
\text { predicted })^{c}\end{array}$ & $\begin{array}{l}\text { Tiotropium } 18 \mu \mathrm{g} \text { qd } \\
\text { Placebo (I:I) }\end{array}$ & $\begin{array}{l}\text { Study treatment given in } \\
\text { addition to LABA or LABA/ICS }\end{array}$ \\
\hline $\begin{array}{l}\text { Chan et al } \\
(2007)^{21}\end{array}$ & 48 weeks & 913 & $\begin{array}{l}39(\leq 65 \\
\text { predicted })^{c}\end{array}$ & $\begin{array}{l}\text { Tiotropium I8 } \mu \mathrm{g} \text { qd } \\
\text { Placebo }(2: 1)\end{array}$ & $\begin{array}{l}\text { Study treatment given in } \\
\text { addition to } L A B A \text { or } L A B A / I C S\end{array}$ \\
\hline $\begin{array}{l}\text { Tashkin } \\
\text { et al }(2008) \\
(\text { UPLIFT })^{22}\end{array}$ & 4 years & 5,993 & 48 (GOLD II-IV) & $\begin{array}{l}\text { Tiotropium I8 } \mu \mathrm{g} \text { qd } \\
\text { Placebo }(1: 1)\end{array}$ & $\begin{array}{l}\text { Study treatment given in } \\
\text { addition to } L A B A \text { or } L A B A / I C S\end{array}$ \\
\hline $\begin{array}{l}\text { Troosters et al } \\
(2014)^{23}\end{array}$ & 24 weeks & 457 & 66 (GOLD II) & $\begin{array}{l}\text { Tiotropium } 18 \mu \mathrm{g} \text { qd } \\
\text { Placebo }(1: 1)\end{array}$ & $\begin{array}{l}\text { OCS (up to } 2 \text { weeks for acute } \\
\text { exacerbations) }\end{array}$ \\
\hline \multicolumn{6}{|c|}{ Tiotropium (via Respimat ${ }^{\circledR}$ device) } \\
\hline $\begin{array}{l}\text { Bateman et al } \\
(2010)^{24}\end{array}$ & I year $\times 2^{b}$ & 1,990 & $45-47$ & $\begin{array}{l}\text { Tiotropium } 5 \mu \mathrm{g} \text { qd } \\
\text { Tiotropium } 10 \mu \mathrm{g} \text { qd } \\
\text { Placebo (I:I:I) }\end{array}$ & ICS/OCS; theophylline \\
\hline $\begin{array}{l}\text { Bateman et al } \\
(2010)^{25}\end{array}$ & I year & 3,991 & 45 & $\begin{array}{l}\text { Tiotropium } 5 \mu \mathrm{g} \text { qd } \\
\text { Placebo (I:I) }\end{array}$ & $\begin{array}{l}\text { Study treatment given in } \\
\text { addition to } L A B A \text { or } L A B A / I C S\end{array}$ \\
\hline \multicolumn{6}{|c|}{ Glycopyrronium } \\
\hline $\begin{array}{l}\text { D'Urzo } \\
\text { et al }(20 \mathrm{II}) \\
(\mathrm{GLOWI})^{26}\end{array}$ & 26 weeks & 822 & $\begin{array}{l}\text { 54-55 } \\
\text { (GOLD II-IV) }\end{array}$ & $\begin{array}{l}\text { Glycopyrronium } 50 \mu \mathrm{g} \text { qd } \\
\text { Placebo (2:1) }\end{array}$ & $\begin{array}{l}\text { ICS at stable dose; OCS and/or } \\
\text { SABA for exacerbations }\end{array}$ \\
\hline $\begin{array}{l}\text { Kerwin } \\
\text { et al }(2012) \\
(\text { GLOW } 2)^{27}\end{array}$ & 52 weeks & $\mathrm{I}, 066$ & 56 (GOLD II-IV) & $\begin{array}{l}\text { Glycopyrronium } 50 \mu \mathrm{g} \text { qd } \\
\text { Tiotropium } 18 \mu \mathrm{g} \text { qd OL } \\
\text { Placebo (2:I:I) }\end{array}$ & $\begin{array}{l}\text { ICS at stable dose; OCS and/or } \\
\text { SABA for exacerbations }\end{array}$ \\
\hline \multicolumn{6}{|l|}{ Umeclidinium } \\
\hline $\begin{array}{l}\text { Donohue et al } \\
(2013)^{28}\end{array}$ & 24 weeks & 1,532 & $\begin{array}{l}47-48 \\
(G O L D ~ I I-I V)\end{array}$ & $\begin{array}{l}\text { Umeclidinium/vilanterol } 62.5 / 25 \mu \mathrm{g} \text { qd } \\
\text { Umeclidinium } 62.5 \mu \mathrm{g} \text { qd } \\
\text { Vilanterol } 25 \mu \mathrm{g} \text { qd } \\
\text { Placebo (3:3:3:2) }\end{array}$ & ICS at stable dose \\
\hline \multicolumn{6}{|l|}{ Aclidinium } \\
\hline $\begin{array}{l}\text { Jones } \\
\text { et al }(2012) \\
(\text { ATTAIN })^{29}\end{array}$ & 24 weeks & 828 & 57 (GOLD II/III) & $\begin{array}{l}\text { Aclidinium } 200 \mu g \text { bid } \\
\text { Aclidinium } 400 \mu g \text { bid } \\
\text { Placebo (I:I:I) }\end{array}$ & $\begin{array}{l}\text { ICS; theophylline; OCS; oxygen } \\
\text { therapy }\end{array}$ \\
\hline $\begin{array}{l}\text { Singh } \\
\text { et al }(20 \mid 4) \\
(\text { ACLIFORM })^{30}\end{array}$ & 24 weeks & 1,729 & 54 (GOLD II/III) & $\begin{array}{l}\text { Aclidinium/formoterol } 400 / 12 \mu \mathrm{g} \text { bid } \\
\text { Aclidinium/formoterol } 400 / 6 \mu \mathrm{g} \text { bid } \\
\text { Aclidinium } 400 \mu \mathrm{g} \\
\text { Formoterol } 12 \mu \mathrm{g} \text { bid } \\
\text { Placebo }(2: 2: 2: 2: 1)\end{array}$ & $\begin{array}{l}\text { ICS; theophylline; OCS; oxygen } \\
\text { therapy }\end{array}$ \\
\hline $\begin{array}{l}\text { D’Urzo } \\
\text { et al }(20 \mid 4) \\
(\text { AUGMENT })^{3 !}\end{array}$ & 24 weeks & $\mathrm{I}, 692$ & $53-55$ & $\begin{array}{l}\text { Aclidinium/formoterol } 400 / 12 \mu \mathrm{g} \text { bid } \\
\text { Aclidinium/formoterol } 400 / 6 \mu \mathrm{g} \text { bid } \\
\text { Aclidinium } 400 \mu \mathrm{g} \text { bid } \\
\text { Formoterol } 12 \mu \mathrm{g} \text { bid } \\
\text { Placebo (I:I:I:1:I) }\end{array}$ & $\begin{array}{l}\text { Stable doses of theophylline, } \\
\text { ICS, or systemic } \\
\text { corticosteroids permitted }\end{array}$ \\
\hline
\end{tabular}

Notes: In all studies, use of other anticholinergics/muscarinic antagonists was not permitted. ${ }^{\text {Postbronchodilator FEV }}$, unless otherwise stated. ${ }^{\mathrm{b} T \text { To }}$ I-year studies were evaluated. 'Reference does not specify if this is pre- or postbronchodilator FEV.

Abbreviations: bid, twice daily; FEV , forced expiratory volume in I second; GOLD, Global initiative for chronic Obstructive Lung Disease; ICS, inhaled corticosteroid; LABA, long-acting $\beta_{2}$-agonist; LAMA, long-acting muscarinic antagonist; OCS, oral corticosteroids; OL, open label; qd, once daily; SABA, short-acting $\beta_{2}$-agonist.

treatment) provides a valid and clinically relevant measure of the activity of a bronchodilator across a 12 or 24 hours' period. A $100 \mathrm{~mL}$ change in $\mathrm{FEV}_{1}$ has been reported as the minimum clinically important difference (MCID) at which patients can perceive a difference in lung function. ${ }^{37}$ Trough $\mathrm{FEV}_{1}$ was evaluated in all 14 of the studies identified in the literature search (Table 2). ${ }^{18-31}$ LAMA monotherapy increased trough $\mathrm{FEV}_{1}$ by $\sim 100 \mathrm{~mL}$ compared with placebo. 
Table 2 Lung function in patients receiving LAMA therapy, compared with placebo, in double-blind, randomized, placebo-controlled studies $\geq 6$ months in duration

\begin{tabular}{|c|c|c|c|c|}
\hline \multirow[t]{2}{*}{ Study } & \multicolumn{2}{|c|}{ FEV, at baseline $(\mathrm{mL})$} & \multicolumn{2}{|l|}{ Trough FEV $(\mathrm{mL})$} \\
\hline & Treatment & Placebo & $\Delta$ vs placebo (at time point) & $P$-value \\
\hline \multicolumn{5}{|c|}{ Tiotropium I 8 $\mu$ g qd (via HandiHaler ${ }^{\circledR}$ device) } \\
\hline Casaburi et al $(2002)^{18}$ & 1,000 & 1,040 & $120-150^{\mathrm{a}}$ (49 weeks) & $<0.01$ \\
\hline Donohue et al $(2002)^{19}$ & 1,110 & 1,060 & 137 (24 weeks) & $<0.000$ I \\
\hline Niewoehner et al $(2005)^{20}$ & 1,040 & 1,040 & 100 (6 months) & $<0.001$ \\
\hline Chan et al $(2007)^{21}$ & 970 & 960 & $100(48 \text { weeks })^{\mathrm{a}}$ & $<0.000$ I \\
\hline Tashkin et al (2008) (UPLIFT) $)^{22}$ & 1,330 & 1,332 & $\begin{array}{l}87-103 \text { (over study period to } \\
48 \text { months) }\end{array}$ & $<0.001$ \\
\hline Troosters et al $(2014)^{23}$ & 1,950 & 1,900 & I 40 (week 24) & $<0.001$ \\
\hline \multirow[t]{3}{*}{ Kerwin et al (20I2) (GLOW2) ${ }^{27}$} & 1,500 & $\mathrm{I}, 500$ & 83 (week I2) & $<0.001$ \\
\hline & & & 84 (week 26) & \\
\hline & & & 89 (week 52) & \\
\hline \multicolumn{5}{|c|}{ Tiotropium 5 g qd (via Respimat ${ }^{\circledR}$ device) } \\
\hline Bateman et al $(2010)^{24}$ & 1,066 & 1,058 & $127\left(\right.$ week 48) ${ }^{\mathrm{a}}$ & $<0.0001$ \\
\hline Bateman et al $(2010)^{25}$ & 1,109 & $|| 0 \mid$, & $102\left(\right.$ week 48) ${ }^{\mathrm{a}}$ & $<0.0001$ \\
\hline \multicolumn{5}{|l|}{ Glycopyrronium $50 \mu \mathrm{g}$ qd } \\
\hline \multirow[t]{2}{*}{ D'Urzo et al (20II) (GLOWI ${ }^{26}$} & 1,490 & 1,450 & $105\left(\right.$ week I2) ${ }^{\mathrm{a}}$ & $<0.001$ \\
\hline & & & II3 (week 26) & \\
\hline \multirow[t]{3}{*}{ Kerwin et al (20I2) (GLOW2) ${ }^{27}$} & $\mathrm{I}, 500$ & $\mathrm{I}, 500$ & $97\left(\right.$ week I2) ${ }^{\mathrm{a}}$ & $<0.001$ \\
\hline & & & 134 (week 26) & \\
\hline & & & 108 (week 52) & \\
\hline \multicolumn{5}{|l|}{ Umeclidinium $62.5 \mu \mathrm{g}$ qd } \\
\hline Donohue et al $(2013)^{28}$ & NR & NR & $115($ day 169) & $<0.001$ \\
\hline \multicolumn{5}{|l|}{ Aclidinium $400 \mu \mathrm{g}$ bid } \\
\hline Jones et al (2012) (ATTAIN) ${ }^{29}$ & 1,510 & $\mathrm{I}, 500$ & $128\left(\right.$ week 24) ${ }^{\mathrm{a}}$ & $<0.000$ I \\
\hline Singh et al (20I4) (ACLIFORM) $)^{30}$ & 1,400 & 1,420 & II7 (week 24) & $<0.001$ \\
\hline D’Urzo et al (20I4) (AUGMENT) $)^{31}$ & 1,340 & $\mathrm{I}, 350$ & $\sim 10 \mathrm{I}($ week 24) & $<0.0001$ \\
\hline
\end{tabular}

Notes: Results for FEV, are expressed as active treatment minus placebo values. All differences vs placebo are statistically significant unless otherwise indicated. ${ }^{\text {PPimary }}$ endpoint. 'bstimated from figure.

Abbreviations: bid, twice daily; $\mathrm{FEV}_{1}$, forced expiratory volume in I second; LAMA, long-acting muscarinic antagonist; NR, not reported; qd, once daily.

Postbronchodilator $\mathrm{FEV}_{1}$ (based on spirometry measurements, commonly 30-45 minutes postadministration of a short-acting bronchodilator) is used for assessing the severity of airflow limitation in patients with COPD. ${ }^{1,22,38}$ In the UPLIFT (Understanding Potential Long-Term Impacts on Function with Tiotropium) study, the rate of decline in postbronchodilator $\mathrm{FEV}_{1}$ was $40 \pm 3 \mathrm{~mL}$ in the tiotropium group and $47 \pm 3$ in the placebo group $(P=0.046)$, suggesting an improvement in airflow limitation in the LAMA group compared with placebo. ${ }^{22}$

Measures of hyperinflation can also provide evidence for the efficacy of bronchodilators. ${ }^{39,40}$ Increases in inspiratory capacity (IC) may be a more sensitive marker for improvements in hyperinflation than $\mathrm{FEV}_{1}$ in patients with moderatesevere COPD. ${ }^{39,40}$ One study found that tiotropium achieved sustained reductions in lung hyperinflation, measured by lung volume and IC, at rest and during exercise. ${ }^{41}$ In addition, use of tiotropium resulted in increases in IC, improving the effects of breathlessness and increasing exercise endurance in stable patients with COPD. ${ }^{41}$ Furthermore, improvements in exercise tolerance accompanied by sustained reductions in lung hyperinflation signified by improvements in IC were seen in moderate-to-severe COPD patients treated with GLY and aclidinium..$^{42,43}$

\section{Exacerbations}

GOLD 2018 defines an exacerbation as "an acute worsening of respiratory symptoms that results in additional therapy". ${ }^{1}$ In clinical trials, the severity of an exacerbation is often determined by the level of management required, eg, patient self-management (mild), treatment with antibiotics/oral corticosteroids (moderate), or emergency room attendance/ hospitalization (severe). ${ }^{44}$ The rate of mild exacerbations recorded may vary between studies due to the method used to record patient symptoms, such as patient diaries ${ }^{45}$ and the EXAcerbations of Chronic pulmonary disease Tool for Patient-Reported Outcomes (EXACT-PRO). ${ }^{14}$ Furthermore, the rate of severe exacerbations as defined by 
hospital/emergency room admission may reflect variations in disease management seen within health care systems; for example, a 10-fold variation in hospital admission rates for COPD has been reported between European countries. ${ }^{45,46}$

Accurate assessment of differences in exacerbations requires appropriately powered studies of suitably long duration ( $\geq 1$ year); often, data with newer LAMAs are from shorter studies powered for efficacy endpoints such as trough $\mathrm{FEV}_{1} \cdot{ }^{19-21,23,26,28-31}$ Endpoints used to assess drug effects may differ between studies; exacerbation rates ${ }^{19,21,23,29,30}$ and timeto-first exacerbation ${ }^{18,20,22,24-28}$ are commonly used to assess differences between treatment arms. Differential dropout rates between treatment arms can result in a loss of statistical power to detect differences in event rates; however, analysis by "time-to-first event" avoids this problem and provides a truer indication of drug effect on exacerbations.

Exacerbation rates were evaluated in 11 of the 14 studies identified (Table 3). ${ }^{18-31}$ Although the overall rate

Table 3 Effect on exacerbations in patients receiving LAMA therapy, compared with placebo, in double-blind, randomized, placebocontrolled studies $\geq 6$ months in duration

\begin{tabular}{|c|c|c|c|c|c|c|c|c|}
\hline \multirow[t]{2}{*}{ Study } & \multicolumn{3}{|c|}{$\begin{array}{l}\text { Annual exacerbation rate (treatment } \\
\text { vs placebo) }\end{array}$} & \multicolumn{3}{|c|}{$\%$ patients with $\geq I$ exacerbation } & \multirow{2}{*}{$\begin{array}{l}\text { Risk reduction } \\
\text { in time to first } \\
\text { exacerbation } \\
\text { (P-value) }\end{array}$} & \multirow[t]{2}{*}{$\begin{array}{l}\text { Exacerbation } \\
\text { type }\end{array}$} \\
\hline & Placebo & Treatment & $\begin{array}{l}\text { Treatment vs } \\
\text { placebo ( } P \text {-value })\end{array}$ & Placebo & Treatment & $\begin{array}{l}\text { Treatment vs } \\
\text { placebo ( } P \text {-value) }\end{array}$ & & \\
\hline \multicolumn{9}{|c|}{ Tiotropium (via HandiHaler ${ }^{\circledast}$ device) } \\
\hline $\begin{array}{l}\text { Casaburi et al } \\
(2002)^{18}\end{array}$ & 0.95 & 0.76 & $\begin{array}{l}20 \% \text { reduction } \\
(0.045)\end{array}$ & 42 & 36 & $\begin{array}{l}14 \% \text { reduction } \\
(<0.05)\end{array}$ & NR $(0.01 \mathrm{I})$ & All \\
\hline $\begin{array}{l}\text { Donohue et al } \\
(2002)^{19}\end{array}$ & NR & NR & NR & NR & NR & NR & NR & NR \\
\hline $\begin{array}{l}\text { Niewoehner et al } \\
(2005)^{20}\end{array}$ & 1.05 & 0.85 & NR $(0.031)$ & 32 & 28 & $\mathrm{OR}=0.8 \mathrm{I}^{\mathrm{a}}(0.037)$ & $17 \%(0.028)$ & $\begin{array}{l}\text { Moderate or } \\
\text { severe }\end{array}$ \\
\hline Chan et al $(2007)^{21}$ & 0.92 & 0.88 & $\mathrm{NR}(\mathrm{NS})$ & 41 & 44 & NR (NS) & NR & Moderate \\
\hline $\begin{array}{l}\text { Tashkin et al } \\
\text { (2008) (UPLIFT) } 22\end{array}$ & 0.85 & 0.73 & $\begin{array}{l}\text { Relative risk }=0.86 \\
(<0.00 \mathrm{I})\end{array}$ & 68 & 67 & NR (NS) & $\mathrm{I} 4 \%(<0.00 \mathrm{I})$ & Moderate \\
\hline $\begin{array}{l}\text { Troosters et al } \\
(2014)^{23}\end{array}$ & NR & NR & NR & NR & NR & NR & NR & \\
\hline $\begin{array}{l}\text { Kerwin et al (2012) } \\
(\text { GLOW2) }\end{array}$ & NR & NR & NR (NS) & NR & NR & $\mathrm{RR}=0.80(\mathrm{NS})$ & $39 \%(0.00 I)$ & $\begin{array}{l}\text { Moderate or } \\
\text { severe }\end{array}$ \\
\hline \multicolumn{9}{|c|}{ Tiotropium (via Respimat ${ }^{\circledR}$ device) } \\
\hline $\begin{array}{l}\text { Bateman et al } \\
(2010)^{b, 24}\end{array}$ & 1.91 & 0.93 & NR (NS) & 44 & 37 & $\mathrm{OR}=0.75(<0.01)$ & $\mathrm{NR}(<0.000 \mathrm{I})$ & Moderate \\
\hline $\begin{array}{l}\text { Bateman et al } \\
(2010)^{25}\end{array}$ & 0.87 & 0.69 & $\begin{array}{l}\text { Relative rate }=0.79 \\
(<0.000 \mathrm{I})\end{array}$ & 43 & 35 & $\begin{array}{l}\mathrm{HR}=0.69 \\
(<0.000 \mathrm{I})\end{array}$ & $31 \%{ }^{\mathrm{a}}(<0.000 \mathrm{I})$ & $\begin{array}{l}\text { Mild or } \\
\text { moderate }\end{array}$ \\
\hline \multicolumn{9}{|c|}{ Glycopyrronium $50 \mu \mathrm{g}$ qd } \\
\hline $\begin{array}{l}\text { D'Urzo et al (20II) } \\
(\mathrm{GLOWI})^{26}\end{array}$ & 0.59 & 0.43 & $\mathrm{RR}=0.72(\mathrm{NS})$ & 24 & 18 & NR & $31 \%(0.023)$ & $\begin{array}{l}\text { Moderate or } \\
\text { severe }\end{array}$ \\
\hline & NR & NR & NR & NR & NR & NR & $65 \%(0.022)$ & Severe \\
\hline $\begin{array}{l}\text { Kerwin et al (20I2) } \\
(\text { GLOW2) }\end{array}$ & NR & NR & NR & 0.80 & 0.54 & $\mathrm{RR}=0.66(0.003)$ & $34 \%(0.001)$ & $\begin{array}{l}\text { Moderate or } \\
\text { severe }\end{array}$ \\
\hline \multicolumn{9}{|c|}{ Umeclidinium $62.5 \mu \mathrm{g} \mathrm{qd}$} \\
\hline $\begin{array}{l}\text { Donohue et al } \\
(2013)^{28}\end{array}$ & NR & NR & NR & 13 & $\begin{array}{l}\mathrm{NR}, 7 \%-9 \% \text { in } \\
\text { active groups }\end{array}$ & NR (NR) & $\sim 40 \%(<0.05)$ & $\begin{array}{l}\text { Moderate or } \\
\text { severe }\end{array}$ \\
\hline \multicolumn{9}{|c|}{ Aclidinium $400 \mu \mathrm{g}$ bid } \\
\hline $\begin{array}{l}\text { Jones et al (2012) } \\
(\text { ATTAIN) })^{29}\end{array}$ & 0.47 & 0.34 & $\mathrm{RR}=0.72(\mathrm{NS})$ & NR & NR & NR & NR & $\begin{array}{l}\text { Moderate or } \\
\text { severe }\end{array}$ \\
\hline & 0.60 & 0.40 & $\mathrm{RR}=0.67(<0.05)$ & NR & NR & NR & NR & All \\
\hline $\begin{array}{l}\text { Singh et al }(2014) \\
(\text { ACLIFORM })^{30}\end{array}$ & 0.36 & 0.29 & $N R(N S)$ & NR & NR & NR & NR & All (HCRU) \\
\hline $\begin{array}{l}\text { D’Urzo et al (2014) } \\
(\text { AUGMENT) })^{3 !}\end{array}$ & NR & NR & NR & NR & NR & NR & NR & \\
\hline
\end{tabular}

Notes: Mild exacerbation defined as increase in symptoms for $\geq 2$ days resulting in an increased use of short-acting bronchodilators and/or ICS. Moderate exacerbation defined as new/increased cough, sputum, sputum purulence, dyspnea, wheeze, or chest discomfort for $\geq 3$ days requiring antibiotics and/or systemic steroids. Severe exacerbations defined as exacerbations leading to hospitalization. HCRU, Healthcare Resource Utilization (defined as an increase of COPD symptoms during $\geq 2$ consecutive

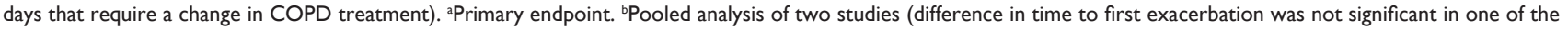
two studies).

Abbreviations: bid, twice daily; HR, hazard ratio; ICS, inhaled corticosteroid; LAMA, long-acting muscarinic antagonist; NR, not reported; NS, not statistically significant; $\mathrm{OR}$, odds ratio; qd, once daily; $R R$, rate ratio. 
of exacerbations was low (generally $<1$ per year in placebo recipients), statistically significant reductions were reported. In three of the four studies in which exacerbations were assessed, tiotropium (HandiHaler ${ }^{\circledR}$ ) significantly reduced the rate of moderate-to-severe exacerbations and all (mild, moderate, or severe) exacerbations compared with placebo, ${ }^{18,20,22}$ while in the remaining study, no significant reduction in moderate-to-severe exacerbations was observed. ${ }^{21}$ Tiotropium (Respimat ${ }^{\circledR}$ ) significantly reduced the rate of moderate-to-severe exacerbations compared with placebo in two studies. ${ }^{24,25}$

Similarly, GLY significantly decreased the risk of first moderate-to-severe exacerbation compared with placebo in both GLOW1 (Glycopyrronium Bromide in COPD AirWays) and GLOW2 studies and significantly reduced the rate of moderate-to-severe exacerbations in GLOW2. ${ }^{26,27}$ Umeclidinium also significantly reduced the risk of timeto-first moderate or severe exacerbation compared with placebo, although the study did not report on exacerbation rate. ${ }^{28}$ Aclidinium significantly reduced all exacerbations vs placebo in ATTAIN (Aclidinium to Treat Airway Obstruction in COPD Patients) but had no significant effect on the frequency of moderate-to-severe exacerbations in ATTAIN or ACLIFORM. ${ }^{29,30}$ Finally, aclidinium significantly reduced the rate of moderate-to-severe exacerbations compared with placebo in a pooled analysis of data from ATTAIN and Aclidinium in Chronic Obstructive Respiratory Disease (ACCORD). ${ }^{47}$ Overall, evidence indicates that LAMAs are effective at reducing the risk of exacerbations in COPD patients.

\section{Dyspnea}

Dyspnea is the fundamental symptom of COPD. ${ }^{1}$ Tools to measure dyspnea include the transition dyspnea index (TDI), which is widely used in clinical trials. ${ }^{48,49}$ TDI measures changes from a baseline state of dyspnea severity in three categories (functional impairment, magnitude of task, and magnitude of effort) and sums to give a score of 0-12 points, with a lower score indicating a greater deterioration in dyspnea severity. ${ }^{49} \mathrm{~A} \geq 1$-unit change in TDI score is defined as the MCID. ${ }^{50}$

TDI was measured in nine of the 14 studies (Table 4). ${ }^{18-31}$ LAMA significantly reduced TDI, with more patients responding to therapy and attaining the MCID vs placebo. ${ }^{18,19,24,26-31}$ These findings indicate that LAMAs are effective at reducing breathlessness that patients are likely to perceive as beneficial.

\section{Health status}

St George's Respiratory Questionnaire (SGRQ) is one of the most widely used tools to assess health status in patients with respiratory disease. SGRQ measures the following three components: frequency and severity of respiratory symptoms, activities that cause dyspnea and are limited by dyspnea, and an "impacts" section, covering aspects of employment, loss of control, expectations, and disturbance to daily life..$^{51}$ Total scores range between 0 and 100 units; higher scores indicate a greater impact on health status. A change in the score of $\geq 4$, compared with either baseline (when assessing an individual's response to treatment) or placebo (for treatment efficacy evaluation in clinical trials), is established as the MCID. ${ }^{51-53}$

Health status was assessed using SGRQ in 11 of the 14 studies (Table 3). ${ }^{18-31}$ Compared with placebo, treatment with tiotropium or GLY resulted in numerical improvements in health status in eight studies; however, differences in SGRQ total score were between -2.7 and -3.5 units and were less than the MCID. ${ }^{18,19,21,22,24-27}$ Umeclidinium significantly improved SGRQ total score from baseline and exceeded the MCID compared with placebo (-4.69). ${ }^{28}$ Aclidinium significantly improved health status from baseline by a similar amount vs placebo in both ATTAIN and AUGMENT (-4.6 and -4.2 , respectively); ${ }^{29,31}$ however, no significant difference was observed in ACLIFORM. ${ }^{30}$ Authors of ACLIFORM attributed a high placebo effect to the lack of significance seen despite the large change from baseline in SGRQ score ( -5.8 to -8.3 units with regimens containing aclidinium vs -6.5 units with placebo).$^{30}$ In general, LAMAs showed an improvement in SGRQ total score compared with placebo, with several studies demonstrating clinically relevant differences.

\section{Safety}

The frequency of adverse events (AEs) was similar between LAMA and placebo in seven of the 14 studies, ${ }^{18,20,24-26,29,30}$ with few observed differences in the incidence of individual AEs (excluding typical anticholinergic AEs). Compared with placebo, a lower frequency of serious AEs occurred with GLY (12.6 vs $16.0 \%){ }^{27}$ whereas a higher frequency was observed with tiotropium (HandiHaler ${ }^{\circledR}$ ) in two studies (18.4 vs $14.1 \%$ and 51.6 vs $50.2 \%),{ }^{21,22}$ umeclidinium in one study (6.0 vs $3.0 \%),{ }^{28}$ and aclidinium in one study (5.0 vs $3.6 \%) .{ }^{31}$ There were no observed differences in mortality between LAMA and placebo.

Anticholinergic AEs include dry mouth, constipation, dysuria, and urinary tract infections. ${ }^{1}$ While few studies 
Table 4 Dyspnea, health status, and rescue medication use in patients receiving LAMA therapy, compared with placebo, in double-blind, randomized, placebo-controlled studies $\geq 6$ months in duration

\begin{tabular}{|c|c|c|c|c|c|c|}
\hline \multirow[t]{2}{*}{ Study } & \multirow[t]{2}{*}{ Treatments } & \multicolumn{2}{|c|}{ TDI focal score } & \multicolumn{2}{|c|}{ Health status (SGRQ) } & \multirow{2}{*}{$\begin{array}{l}\text { Rescue medication } \\
\text { use (treatment vs } \\
\text { placebo) }\end{array}$} \\
\hline & & $\begin{array}{l}\Delta \text { vs } \\
\text { placebo }\end{array}$ & $\begin{array}{l}\text { Responders (\%) } \\
\text { (treatment vs } \\
\text { placebo) }\end{array}$ & $\begin{array}{l}\Delta \text { vs } \\
\text { placebo }\end{array}$ & $\begin{array}{l}\text { Responders (\%) } \\
\text { (treatment vs } \\
\text { placebo) }\end{array}$ & \\
\hline \multicolumn{7}{|c|}{ Tiotropium (via HandiHaler ${ }^{\circledast}$ device) } \\
\hline $\begin{array}{l}\text { Casaburi et al } \\
(2002)^{18}\end{array}$ & Tiotropium $18 \mu \mathrm{g}$ qd & $\begin{array}{l}0.8-1.1 \\
P<0.001\end{array}$ & $\begin{array}{l}42-47 \text { vs } 29-34 \\
P<0.00 \text { I }\end{array}$ & $\begin{array}{l}N R \\
P<0.05\end{array}$ & $\begin{array}{l}49 \text { vs } 30 \\
\text { Significant but } \\
P \text {-value not given }\end{array}$ & $\begin{array}{l}3.2 \text { vs } 4.1 \text { doses/day } \\
P<0.0 \text { I }\end{array}$ \\
\hline $\begin{array}{l}\text { Donohue et al } \\
(2002)^{19}\end{array}$ & Tiotropium $18 \mu \mathrm{g}$ qd & $\begin{array}{l}1.02 \\
P=0.01\end{array}$ & $\begin{array}{l}42 \text { vs } 26 \\
P<0.01\end{array}$ & $\begin{array}{l}-2.71 \\
P<0.05\end{array}$ & $\begin{array}{l}51 \text { vs } 42 \\
P<0.05\end{array}$ & $\begin{array}{l}-1.45 \text { puffs/day vs placebo } \\
P<0.000 \text { I }\end{array}$ \\
\hline $\begin{array}{l}\text { Niewoehner et al } \\
(2005)^{20}\end{array}$ & Tiotropium $18 \mu \mathrm{g}$ qd & NR & NR & NR & NR & NR \\
\hline Chan et al $(2007)^{21}$ & Tiotropium $18 \mu \mathrm{g}$ qd & NR & NR & $\begin{array}{l}-2.8 \\
P<0.01\end{array}$ & $\begin{array}{l}53 \text { vs } 44 \\
\text { NS }\end{array}$ & $\begin{array}{l}\sim 6 \text { fewer puffs/week vs } \\
\text { placebo } \\
P<0.0 \text { I }\end{array}$ \\
\hline $\begin{array}{l}\text { Tashkin et al } \\
(2008)(\text { UPLIFT) })^{22}\end{array}$ & Tiotropium I $8 \mu \mathrm{g}$ qd & NR & NR & $\begin{array}{l}-2.7 \\
P<0.001\end{array}$ & $\begin{array}{l}45-49 \text { vs } 36-41 \\
P<0.00 \text { I }\end{array}$ & NR \\
\hline $\begin{array}{l}\text { Troosters et al } \\
(2014)^{23}\end{array}$ & Tiotropium I $8 \mu \mathrm{g}$ qd & NR & NR & NR & NR & NR \\
\hline Kerwin et al & Tiotropium I $8 \mu \mathrm{g}$ qd OL & & & & & \\
\hline \multirow[t]{2}{*}{$(2012)(G L O W 2)^{27}$} & Week 26 & $\begin{array}{l}0.94 \\
P=0.002\end{array}$ & $\begin{array}{l}53.4 \text { vs } 44.2 \\
P=0.032\end{array}$ & $\begin{array}{l}-2.52 \\
P<0.05\end{array}$ & NR & NR \\
\hline & Week 52 & $\begin{array}{l}0.66 \\
P=0.037\end{array}$ & NR & $\begin{array}{l}-2.84 \\
P=0.014\end{array}$ & $\begin{array}{l}59 \text { vs } 5 I \\
\text { NS }\end{array}$ & $\begin{array}{l}-0.63 \text { puffs/day } \\
P<0.0 \text { I }\end{array}$ \\
\hline \multicolumn{7}{|c|}{ Tiotropium (via Respimat ${ }^{\circledR}$ device) } \\
\hline $\begin{array}{l}\text { Bateman et al } \\
(2010)^{24}\end{array}$ & Tiotropium $5 \mu \mathrm{g}$ qd & $\begin{array}{l}1.05 \\
P<0.0001\end{array}$ & $\begin{array}{l}56 \text { vs } 44 \\
P<0.000 \text { I }\end{array}$ & $\begin{array}{l}-3.5 \\
P<0.0001\end{array}$ & $\begin{array}{l}5 \text { I vs } 4 \text { I } \\
P<0.05\end{array}$ & $\begin{array}{l}-0.6 \text { occasions/day } \\
P<0.000 \text { I }\end{array}$ \\
\hline $\begin{array}{l}\text { Bateman et al } \\
(2010)^{25}\end{array}$ & Tiotropium $5 \mu \mathrm{g} q d$ & NR & NR & $\begin{array}{l}-2.9 \\
P<0.0001\end{array}$ & $\begin{array}{l}50 \text { vs } 4 I \\
P<0.000 I\end{array}$ & NR \\
\hline \multicolumn{7}{|l|}{ Glycopyrronium } \\
\hline $\begin{array}{l}\text { D'Urzo et al } \\
\text { (20II) }(\mathrm{GLOWI})^{26}\end{array}$ & Glycopyrronium $50 \mu \mathrm{g}$ qd & $\begin{array}{l}1.04 \\
P<0.001\end{array}$ & $\begin{array}{l}6 I \text { vs } 48 \\
P=0.001\end{array}$ & $\begin{array}{l}-2.8 \\
P=0.004\end{array}$ & $\begin{array}{l}57 \text { vs } 46 \\
P=0.006\end{array}$ & $\begin{array}{l}-0.46 \text { puffs } / \text { day } \\
P=0.005\end{array}$ \\
\hline Kerwin et al & Glycopyrronium $50 \mu \mathrm{g}$ qd & & & & & \\
\hline \multirow[t]{2}{*}{$(2012)(G L O W 2)^{27}$} & Week 26 & $\begin{array}{l}0.81 \\
P=0.002\end{array}$ & $\begin{array}{l}55 \text { vs } 44 \\
P=0.01\end{array}$ & $\begin{array}{l}-3.38 \\
P<0.001\end{array}$ & NR & NR \\
\hline & Week 52 & $\begin{array}{l}0.57 \\
P=0.038\end{array}$ & NR & $\begin{array}{l}-3.32 \\
P<0.001\end{array}$ & $\begin{array}{l}54 \text { vs } 5 I \\
\text { NS }\end{array}$ & $\begin{array}{l}-0.37 \text { puffs/day } \\
P<0.05\end{array}$ \\
\hline \multicolumn{7}{|l|}{ Umeclidinium } \\
\hline $\begin{array}{l}\text { Donohue et al } \\
(20 \mid 3)^{28}\end{array}$ & Umeclidinium $62.5 \mu \mathrm{g}$ qd & $\begin{array}{l}1.0 \\
P \leq 0.001\end{array}$ & $\begin{array}{l}53 \text { vs } 4 \mathrm{I} \\
P \leq 0.0 \mathrm{I}\end{array}$ & $\begin{array}{l}-4.69 \\
P \leq 0.001\end{array}$ & $\begin{array}{l}44 \text { vs } 34 \\
P \leq 0.01\end{array}$ & $\begin{array}{l}-0.3 \text { puffs/day } \\
\text { NS }\end{array}$ \\
\hline \multicolumn{7}{|l|}{ Aclidinium } \\
\hline $\begin{array}{l}\text { Jones et al }(2012) \\
(\text { ATTAIN })^{29}\end{array}$ & Aclidinium $400 \mu \mathrm{g}$ bid & $\begin{array}{l}1.0 \\
P<0.001\end{array}$ & $\begin{array}{l}57 \text { vs } 46 \\
P<0.0 \text { I }\end{array}$ & $\begin{array}{l}-4.6 \\
P<0.0001\end{array}$ & $\begin{array}{l}57 \text { vs } 4 \text { I } \\
P<0.00 \text { I }\end{array}$ & $\begin{array}{l}-0.95 \text { puffs } / \text { day } \\
P<0.000 \text { I }\end{array}$ \\
\hline $\begin{array}{l}\text { Singh et al }(2014) \\
(\text { ACLIFORM })^{30}\end{array}$ & Aclidinium $400 \mu \mathrm{g}$ bid & $\begin{array}{l}0.9 \\
P<0.01\end{array}$ & $\begin{array}{l}57 \text { vs } 46 \\
P<0.05\end{array}$ & $\begin{array}{l}0.71 \\
\text { NS }\end{array}$ & $\mathrm{NR}$ & NR \\
\hline $\begin{array}{l}\text { D'Urzo } \\
\text { et al }(20 \mid 4) \\
(\text { AUGMENT })^{31}\end{array}$ & Aclidinium $400 \mu \mathrm{g}$ bid & $\begin{array}{l}0.98^{a} \\
P \leq 0.001\end{array}$ & $\begin{array}{l}55 \text { vs } 37 \\
P \leq 0.001\end{array}$ & $\begin{array}{l}-4.23^{\mathrm{a}} \\
P \leq 0.00 \mathrm{I}\end{array}$ & $\begin{array}{l}55 \text { vs } 39 \\
P \leq 0.001\end{array}$ & $\begin{array}{l}-0.68 \text { from baseline with } \\
\text { aclidinium (placebo NR) } \\
P<0.000 \text { I }\end{array}$ \\
\hline
\end{tabular}

Notes: All comparisons are vs placebo. Responder analyses represent percentages of patients with TDI score $\geq I$ or SGRQ improvement $\geq 4$ points. ${ }^{\text {a }}$ alues are estimated from figures in the associated reference.

Abbreviations: bid, twice daily; LAMA, long-acting muscarinic antagonist; NR, not reported; NS, not statistically significant; OL, open label; qd, once daily; SAL, salmeterol; SGRQ, St George's Respiratory Questionnaire; TDI, transition dyspnea index.

reported anticholinergic AEs, the incidence of dry mouth was reported in some studies, with rates ranging from 1.3 to $16 \%$ with tiotropium (HandiHaler ${ }^{\circledR}$ ), 3.1 to $7.2 \%$ with tiotropium $\left(\right.$ Respimat $\left.^{\circledR}\right)$, and 0.6 to $0.7 \%$ with aclidinium (Table 5 ). ${ }^{18-31}$
In a 52-week extension to the 12-week ACCORD trial, the incidence of dry mouth was similar between patients receiving aclidinium 200 and $400 \mu \mathrm{g} .{ }^{32}$ No specific data were provided for studies of GLY or umeclidinium. Details provided 
Table 5 AEs in patients receiving LAMA therapy, compared with placebo, in double-blind, randomized, placebo-controlled studies $\geq 6$ months in duration

\begin{tabular}{|c|c|c|c|c|}
\hline \multirow[t]{2}{*}{ Study } & \multirow[t]{2}{*}{ Treatments } & \multicolumn{3}{|c|}{ Typical anticholinergic AEs (\% patients) } \\
\hline & & Dry mouth & Constipation & UTI \\
\hline \multicolumn{5}{|c|}{ Tiotropium (via HandiHaler ${ }^{\circledR}$ device) } \\
\hline Casaburi et al $(2002)^{18}$ & Tiotropium I8 $\mu \mathrm{g}$ qd/placebo & $16 / 2.7$ & NR & NR \\
\hline Donohue et al $(2002)^{19}$ & Tiotropium $18 \mu \mathrm{g}$ qd/placebo & IO/NR & NR & NR \\
\hline Niewoehner et al $(2005)^{20}$ & Tiotropium I $8 \mu \mathrm{g}$ qd/placebo & NR & NR & NR \\
\hline Chan et al $(2007)^{21}$ & Tiotropium $18 \mu \mathrm{g}$ qd/placebo & $3.5 / 3.6$ & NR & NR \\
\hline Tashkin et al (2008) (UPLIFT) $)^{22}$ & Tiotropium $18 \mu \mathrm{g}$ qd/placebo & $1.7 / 0.9^{\mathrm{a}}$ & $1.6 / 1.3^{\mathrm{a}}$ & 2. $1 / 2.0^{\mathrm{a}}$ \\
\hline Troosters et al $(2014)^{23}$ & Tiotropium $18 \mu \mathrm{g}$ qd/placebo & $1.3 / 0.9$ & NR & NR \\
\hline Kerwin et al (20I2) (GLOW2)27 & Tiotropium I8 $\mu \mathrm{g}$ qd OL/placebo & $1.5 / 1.9$ & NR & $6.0 / 3.0$ \\
\hline \multicolumn{5}{|l|}{ Tiotropium (via Respimat ${ }^{\circledR}$ device) } \\
\hline Bateman et al $(2010)^{24}$ & Tiotropium $5 \mu \mathrm{g}$ qd/placebo & $7.2 / 2.1$ & NR & NR \\
\hline Bateman et al $(2010)^{25}$ & Tiotropium $5 \mu \mathrm{g}$ qd/placebo & $3.1 / 1.4$ & NR & NR \\
\hline \multicolumn{5}{|l|}{ Glycopyrronium } \\
\hline D’Urzo et al (20II) (GLOWI)26 & Glycopyrronium $50 \mu \mathrm{g} \mathrm{qd/placebo}$ & NR & NR & NR \\
\hline Kerwin et al (20I2) (GLOW2) ${ }^{27}$ & Glycopyrronium $50 \mu \mathrm{g}$ qd/placebo & $3.0 / 1.9$ & NR & $2.7 / 3.0$ \\
\hline \multicolumn{5}{|l|}{ Umeclidinium } \\
\hline Donohue et al $(2013)^{28}$ & Umeclidinium $62.5 \mu \mathrm{g}$ qd/placebo & NR & NR & NR \\
\hline \multicolumn{5}{|l|}{ Aclidinium } \\
\hline Jones et al (20I2) (ATTAIN) ${ }^{29}$ & Aclidinium $400 \mu \mathrm{g}$ bid/placebo & NR & NR & $0.7 / 0.7$ \\
\hline Singh et al (20I4) (ACLIFORM) $)^{30}$ & Aclidinium $400 \mu \mathrm{g}$ bid/placebo & NR & NR & NR \\
\hline D’Urzo et al (20I4) (AUGMENT) $)^{31}$ & Aclidinium $400 \mu \mathrm{g}$ bid/placebo & $0.6 / 0.3$ & $2.1 / 1.8$ & $3.3 / 3.0$ \\
\hline
\end{tabular}

Notes: ancidence rate per 100 patient years.

Abbreviations: AEs, adverse events; bid, twice daily; LAMA, long-acting muscarinic antagonist; NR, not reported; OL, open label; qd, once daily; UTI, urinary tract infection.

in corresponding SmPCs reported dry mouth in 4\% of patients receiving tiotropium (HandiHaler ${ }^{\mathbb{B}}$ ), $2.9 \%$ of patients receiving tiotropium $\left(\right.$ Respimat $\left.^{\circledR}\right)$, and $2.2 \%$ of patients receiving GLY; specific information was not provided in umeclidinium or aclidinium SmPCs. ${ }^{8-11}$ Therefore, LAMA safety profiles appear to be acceptable in the trial patients.

\section{Comparisons between LAMAs}

Results presented here are from separate studies with different settings and designs and are therefore not directly comparable. In the absence of head-to-head studies, comparisons of results from separate studies should be viewed with caution. However, head-to-head comparisons of LAMA therapies have shown some differences, notably the faster onset of action of GLY vs tiotropium. ${ }^{27,54}$ In GLOW5, GLY increased $\mathrm{FEV}_{1}$ by $51 \mathrm{~mL}$ compared with tiotropium at 5 minutes postdose and by $63 \mathrm{~mL}$ at 15 minutes postdose on day $1(P<0.01) .{ }^{54}$ In GLOW2, GLY increased FEV ${ }_{1}$ by $143 \mathrm{~mL}$ vs placebo at 15 minutes postdose $(P<0.001)$ and almost doubled the response seen with tiotropium at that timepoint $(78 \mathrm{~mL})$. Any perceivable benefit observed by the patient following the first dose of medication may be lost following subsequent maintenance doses, ${ }^{55}$ however, may become relevant again when patients do not adhere to daily maintenance treatment, a problem well documented in COPD patients. In a systematic review and network meta-analysis examining the comparative efficacy of LAMAs in COPD, changes from baseline in trough $\mathrm{FEV}_{1}$ at 12 weeks favoring GLY, aclidinium, and umeclidinium vs tiotropium were found; however, the study concluded that these agents had at least comparable efficacy to that of tiotropium. ${ }^{56}$

GLY, tiotropium, and aclidinium had similar effects on measures of lung function, dyspnea, health status, and exacerbations. ${ }^{27,54,57}$ In GLOW2, GLY and tiotropium reduced the risk of first moderate-to-severe exacerbation to a similar extent vs placebo ( 34 and $39 \%$, respectively, both $P=0.001$ ). ${ }^{27}$ GLY significantly reduced the rate of moderate-to-severe exacerbations by $34 \%$ compared with placebo ( 0.54 vs 0.80 per year; rate ratio $[\mathrm{RR}] 0.66 ; 95 \%$ confidence interval [CI] $0.496-0.821 ; P=0.003)$. GLY and open-label tiotropium had similar efficacy in reducing all exacerbations (RR 1.01) and improving lung function and health status in a 64-week study of 1,483 patients with COPD and severe-to-very severe airflow limitation. ${ }^{58,59}$ A network meta-analysis demonstrated aclidinium to be at least comparable to GLY and tiotropium in terms of trough $\mathrm{FEV}_{1}$, health status (assessed by SGRQ with the proportion of responders achieving at least a four point improvement), and dyspnea (assessed by TDI score 
where the majority of responders achieved at least a one point improvement). ${ }^{57}$ In a pooled analysis, aclidinium also reduced the rate of exacerbations of any severity and moderate-tosevere exacerbations by $\sim 20 \%$ compared with placebo, which is consistent with the other LAMAs. ${ }^{60}$

\section{Discussion}

\section{Relevance of the study populations to clinical practice}

The clinical studies reviewed were conducted mostly in patients with moderate-to-severe airflow limitation (GOLD grade II or III), this is likely similar to the patient population in clinical practice. ${ }^{61-64}$ Most studies included a small percentage of patients with very severe airflow limitation (GOLD grade IV) and excluded patients with mild airflow limitation (GOLD grade I) as well as hypoxemia on long-term oxygen therapy. The GOLD 2018 stepwise ABCD treatment approach is based only on symptoms and exacerbation risk (as opposed to former recommendations based on the severity of airflow limitation). ${ }^{1}$

Patients with life-threatening comorbid conditions that could interfere with study results are excluded from clinical studies. ${ }^{65}$ However, physicians can expect to see a range of comorbidities in COPD patients in clinical practice, including cardiovascular disease, anxiety/depression, asthma, diabetes, kidney disease, and chronic pain. ${ }^{12,62}$ One study estimated that $\sim 98 \%$ of COPD patients had $\geq 1$ comorbidity, while $\geq 50 \%$ of them have $\geq 4$ comorbidities. ${ }^{66}$ The DACCORD (Outpatient Care with Long-Acting Bronchodilators: COPD Registry in Germany) ongoing noninterventional study estimated a lower value of 78.3\%. ${ }^{64,66}$ More trials will attempt to include a wider range of patients with COPD to accurately reflect the prevalence of comorbidities in the real world. ${ }^{67}$ Supplementary medications to treat comorbidities increase the risk of unwanted drug interactions and AEs, potentially altering a patient's compliance to treatment. ${ }^{68}$ Therefore, physicians need to remain aware of the above factors and use their judgment to assess the risk-benefit profile of each treatment before arriving at an appropriate decision.

\section{Efficacy of LAMAs in clinical practice}

$\mathrm{FEV}_{1}$ was the most common primary outcome measure of lung function in the studies reviewed, as suggested by the United States Food and Drug Administration (FDA) and EMA. Other patient-reported outcome (PRO) measurements such as breathlessness, health status, and exacerbation provide a robust basis for evaluating the potential benefits of LAMAs.
The data discussed suggest that LAMAs improve lung function, dyspnea, and health status compared with placebo.

COPD patients often reduce their daily activities according to symptom intensity and may not perceive full treatment benefit unless encouraged to increase activity levels. Notably, increased exercise capacity does not necessarily result in enhanced physical activity and some experimental methods are not representative of real-life exercise capacity. ${ }^{69}$

Exacerbations are associated with a poor prognosis in terms of health status, ${ }^{70,71}$ lung function decline, ${ }^{72,73}$ and mortality risk..$^{74,75}$ Therefore, prevention of COPD exacerbations is an important treatment goal. ${ }^{1}$ Among the LAMAs reviewed, GLY and tiotropium had the most evidence supporting a beneficial effect on exacerbations; both significantly prevented or reduced the rate of moderate-to-severe exacerbations compared with placebo in studies of $>1$ year. ${ }^{22,24,25,27}$ Umeclidinium and aclidinium have yet to be assessed in a study of $>24$ weeks and appropriately powered to eliminate seasonal influences and test for differences in exacerbations. ${ }^{28-30}$

LAMA and LABA monotherapies appear to have a similar effect on $\mathrm{FEV}_{1}$ and symptom relief. ${ }^{4}$ POET demonstrated that the LAMA tiotropium was more effective than the LABA salmeterol at reducing exacerbation risk, ${ }^{6}$ which may be due to the presence of patient subgroups with polymorphisms of the $\beta_{2}$-adrenoceptor that affect the response to LABA treatment. ${ }^{76}$ While GOLD recommends LAMAs as a treatment strategy for exacerbation prevention, it gives no treatment preference for symptom relief. ${ }^{1}$

Only one study prospectively evaluated a LAMA as initial (and sole) maintenance therapy in patients with GOLD grade II COPD who were treatment naive. The objective was to evaluate the difference between tiotropium vs placebo on $\mathrm{FEV}_{1}$. Tiotropium significantly improved lung function and PROs compared with placebo. ${ }^{23}$ Other studies permitted the concomitant use of ICS, as suggested by GOLD, ${ }^{1}$ and therefore may not give a clear indication of the efficacy of bronchodilator monotherapy. It is not clear whether ICS treatment was appropriate in these patients since current knowledge suggests that ICS may only be effective in preventing exacerbations in a subgroup of patients with COPD. ${ }^{77}$ However, a pooled analysis of data from two GLY studies showed a similar efficacy of GLY and tiotropium on lung function and exacerbation outcomes in patients regardless of ICS use. ${ }^{78}$

Combining the two classes of bronchodilator results in improvements in lung function, dyspnea, health status, rescue medication use, and exacerbations compared with LAMA monotherapy ${ }^{58,79-84}$ For patients with persistent symptoms 
on monotherapy, LABA/LAMA therapy is recommended while patients with severe symptoms should consider dual bronchodilation as initial treatment. ${ }^{1}$ In BLAZE, IND/GLY significantly improved dyspnea, lung function, and rescue medication use compared with placebo and tiotropium monotherapy in symptomatic patients (modified Medical Research Council [mMRC] dyspnea scale $>2) .{ }^{84}$ In addition, in a post hoc analysis of SHINE, LABA/LAMA significantly improved dyspnea and lung function compared with tiotropium in patients with moderate-to-severe dyspnea. ${ }^{83}$ In patients with a history of $\geq 1$ exacerbation in SPARK, IND/GLY significantly reduced the rate of moderate-tosevere COPD exacerbations compared with GLY and the rate of all exacerbations vs GLY and tiotropium. ${ }^{58}$ INSPIRE reported similar effects on exacerbation reduction for tiotropium and salmeterol/fluticasone propionate (SFC) in patients with a history of exacerbations. ${ }^{85}$ Furthermore, FLAME demonstrated that IND/GLY was significantly more effective than SFC in preventing all and moderate or severe exacerbations in patients with a history of $\geq 1$ exacerbation. ${ }^{86}$ Therefore, GOLD recommendations for ICS/LABA use are limited to an alternative to LABA/LAMA and for patients with features of asthma-COPD overlap (ACO).

LAMAs are often used in conjunction with ICS/LABA, commonly referred to as triple therapy. ${ }^{12}$ Several studies have demonstrated the superiority, in terms of lung function, of combinations of ICS, LABA, and LAMA over ICS/ LABA. ${ }^{14,87}$ If exacerbations persist despite LABA/LAMA or ICS/LABA therapy in GOLD Group D patients, GOLD recommends stepping up to triple therapy. ${ }^{1}$ Indeed, clinical trial evidence supporting this recommendation is beginning to emerge from studies such as TRILOGY (which assessed beclometasone dipropionate, formoterol fumarate, and GLY), FULFIL, and IMPACT (assessing fluticasone furoate, umeclidinium, and vilanterol). ${ }^{14,87,88}$

Studies show ICS providing a small, clinically insignificant increase in lung function $(32-48 \mathrm{~mL})$ when used in combination with LABA compared with LABA monotherapy, ${ }^{78-80}$ but this increase achieved statistical significance $(P<0.001)$ in only one of the three studies. ${ }^{80}$ Similarly, WISDOM (Withdrawal of Inhaled Steroids during Optimized Bronchodilator Management) demonstrated a statistically significant decrease in trough $\mathrm{FEV}_{1}$ following complete ICS withdrawal compared with ICS continuation $(43 \mathrm{~mL}, P=0.001)$ in patients with severe-to-very severe airflow limitation and $\geq 1$ exacerbation in the previous year. ${ }^{89}$ Comparing this with the improvement when one bronchodilator is added to another $(120-168 \mathrm{~mL})$, any benefit seen with triple therapy vs one bronchodilator only may be driven mainly by dual bronchodilation. GOLD recommends ICS for patients with an exacerbation history (GOLD Groups C and D), yet they are prescribed to patients in all GOLD Groups. Further investigation is therefore needed to identify reasons why large numbers of primary care patients have been inappropriately escalated to or initiated on ICS/LABA therapy, to better understand the right population eligible for triple therapy and to elucidate any benefit of ICS over LABA/LAMA in triple therapy, data that are not yet available..$^{90-92}$ If ICS treatment has been inappropriately prescribed, WISDOM demonstrated that ICS can be withdrawn from triple therapy in patients with severe-to-very severe COPD with a low baseline exacerbation rate without an increase in the risk of exacerbation provided adequate bronchodilator therapy is in place. ${ }^{93}$

As GOLD guidance is limited regarding which patients should receive ICS treatment, it has become apparent that phenotyping, subgrouping, and/or endotyping can help tailor the management of a patient with COPD ensuring they get the appropriate treatment they need..$^{94}$ One study found that inflammatory phenotypes, such as frequent exacerbators, patients with chronic bronchitis, and those with a number of comorbidities, needed ICS treatment, ${ }^{94}$ whereas patients with emphysema required dual bronchodilation. ${ }^{94}$ The GesEPOC guidelines also propose treatment approaches based on clinical phenotypes, but without evidence base. ${ }^{15}$ For example, roflumilast is recommended in patients with an exacerbator phenotype and chronic bronchitis, as well as antibiotic therapy guided by sputum purulence in hospitalized patients with COPD exacerbations. ${ }^{15}$

Blood eosinophils may further help determine which patients may benefit from ICS treatment, with post hoc analyses suggesting a greater benefit of ICS/LABA vs LABA monotherapy in patients with elevated blood eosinophils vs those with lower blood eosinophil counts. ${ }^{95-97}$ However, given the nature of available evidence, which arises predominantly from post hoc analyses, ${ }^{86,98}$ although data exist from a prospective analysis of FLAME, eosinophil-guided ICS treatment for COPD exacerbations is currently a controversial area. ${ }^{86,98}$ Using clinical phenotypes and biomarkers as a form of personalized medicine could be highly valuable to clinical practice, as it enables predictions to be made regarding which patients will respond well to certain classes of drugs. ${ }^{99,100}$

\section{Inhaler selection}

Detailed consideration of the characteristics of the different LAMA delivery inhalers is summarized in Table $6 .^{8-11,101-106}$ Given the fact that patients receive intensive education on the 
Table 6 Inhalers used with LAMAs for COPD

\begin{tabular}{|c|c|c|c|c|c|}
\hline $\begin{array}{l}\text { Property of } \\
\text { inhaler }\end{array}$ & HandiHaler ${ }^{\circledR 9,101}$ & Respimat $^{\circledR 8,102,103}$ & Breezhaler ${ }^{\circledR 10,101}$ & Ellipta ${ }^{\circledR 1} 1,104,105$ & Genuair $^{\circledR 9,106}$ \\
\hline $\begin{array}{l}\text { LAMA } \\
\text { administered }\end{array}$ & Tiotropium & Tiotropium & Glycopyrronium & Umeclidinium & Aclidinium \\
\hline $\begin{array}{l}\text { Single dose } \\
\text { or multidose }\end{array}$ & Single dose & Multidose & Single dose & Multidose & Multidose \\
\hline Type & Dry powder & Soft mist (spray) & Dry powder & Dry powder & Dry powder \\
\hline Resistance & High & Low & Low & Medium & Medium \\
\hline $\begin{array}{l}\text { Inhalations } \\
\text { for each use }\end{array}$ & One capsule ${ }^{a}$ & Two actuations & One capsule ${ }^{b}$ & One & One \\
\hline Dose counter & N/A & $\begin{array}{l}\text { Dose indicator } \\
\text { shows approximately } \\
\text { how much left }\end{array}$ & $\mathrm{N} / \mathrm{A}$ & $\begin{array}{l}\text { Yes (on opening } \\
\text { cover) }\end{array}$ & $\begin{array}{l}\text { Dose indicator (counts in } \\
\text { intervals of } 10 \text { ) }\end{array}$ \\
\hline $\begin{array}{l}\text { Confirmation } \\
\text { of dose }\end{array}$ & $\begin{array}{l}\text { No (capsule is } \\
\text { opaque but can } \\
\text { be opened) }\end{array}$ & No & $\begin{array}{l}\text { Yes (hear the click when } \\
\text { the capsule is pierced and } \\
\text { the whirring sound during } \\
\text { inhalation; feel the lactose } \\
\text { in the product; see the } \\
\text { clear/empty capsule) }\end{array}$ & $\begin{array}{l}\text { Yes (clicking sound } \\
\text { when cover is } \\
\text { opened and dose } \\
\text { is ready; counter } \\
\text { counts down by one) }\end{array}$ & $\begin{array}{l}\text { Yes (green control window } \\
\text { confirms that product is ready } \\
\text { for inhalation - this turns back } \\
\text { to red to confirm that full dose } \\
\text { has been taken; clicking sound } \\
\text { signals correct inhalation) }\end{array}$ \\
\hline $\begin{array}{l}\text { Locks when } \\
\text { empty }\end{array}$ & N/A & Yes & $\mathrm{N} / \mathrm{A}$ & No & Yes \\
\hline Refillable & $\begin{array}{l}\text { Yes (clean monthly; } \\
\text { replace after } \\
\text { I year) }\end{array}$ & $\begin{array}{l}\text { No (replace if } \\
\text { empty) }\end{array}$ & $\begin{array}{l}\text { Yes within the same } \\
\text { prescription. Each inhaler } \\
\text { should be replaced after } \\
30 \text { days of use }\end{array}$ & $\begin{array}{l}\text { No (replace if } \\
\text { empty) }\end{array}$ & No (replace if empty) \\
\hline
\end{tabular}

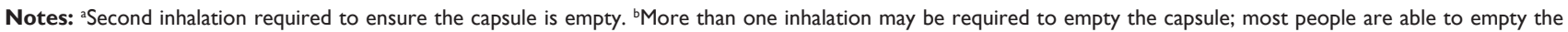
capsule with one or two inhalations.

Abbreviations: LAMA, long-acting muscarinic antagonist; N/A, not available.

use of COPD delivery inhalation devices, randomized controlled trials are not considered suitable for the examination of whether inhaler choice affects clinical outcomes. Nevertheless, one study demonstrated that critical error rates irrespective of inhaler type were associated with severe COPD exacerbations, indicating that training patients in the use of inhaler devices is an essential aspect of treatment efficacy. ${ }^{107}$ The impact of the inhaler on treatment adherence, which may be as poor as $50 \%$ in clinical practice among patients with COPD, should also be considered. ${ }^{108,109}$ Therefore, patient satisfaction with their inhaler is likely to encourage treatment adherence and impact clinical outcomes. ${ }^{110,111}$

A recent study analyzed the relative importance of different attributes of a once-daily dosing inhaler device, determined by patients with $\geq 6$ months COPD: patients cited ease of use, dose delivery recording, and dose capacity (multi- vs single-dose devices) as the most important attributes. ${ }^{112}$ Conjoint analysis indicated that attributes related to device characteristics accounted for $88 \%$ of the relative importance patients placed on the device, whereas the number of doses each day accounted for $12 \%$ of the relative importance. ${ }^{112}$ However, evidence suggests that the majority of patients prefer a once-daily dosing regimen compared with multiple daily dosing, ${ }^{113}$ even if device characteristics are ranked higher in importance. In contrast, frequency of dosing is known to inversely affect treatment adherence. ${ }^{114}$ However, some patients with severe COPD may be familiar with twicedaily treatment regimens and may psychologically prefer this to a once-daily schedule, particularly those suffering from severe morning and nighttime symptoms. Different factors govern inhaler preference in individual patients; if problems seem insurmountable with one inhaler, there should be a low threshold for switching to an alternative LAMA/inhaler.

Internal resistance varies between different inhaler devices and can affect both ease of inhalation and drug deposition. ${ }^{115}$ Data on inhaler resistance are from in vitro studies, and their importance and applicability in certain real-life situations are unclear.

Furthermore, studies have assessed the relative patient preference for currently available dry powder inhalers with variable results. ${ }^{116-119}$ Prescribers should take an active role in teaching their patients the correct inhalation technique for each device at initial assessment. Frequent follow-up and monitoring are critical to ensure that efficacy is sustained, especially in patients with very severe airflow limitation. The significance and benefits of these real-life assessments for physicians and patients in clinical practice outweigh the relevance of experimental data. 
Additionally, for patients receiving treatment with a single bronchodilator, choice of initial therapy may be influenced by the availability of a dual bronchodilation fixeddose combination (FDC) containing the same agent and administered via the same delivery device, should the patient subsequently require additional COPD treatment. Therefore, receiving monotherapy or FDC therapy from a single type of inhaler may be advantageous in terms of convenience and potentially adherence and dosing regimens should be considered to allow the combination of different drugs into the same inhaler to prevent patient confusion.

\section{The importance of distinguishing between asthma and COPD}

Many patients with COPD meet the $\mathrm{FEV}_{1}$ reversibility criteria required for asthma diagnosis. ${ }^{120}$ This may result in disease misclassification if a thorough clinical history is not taken during initial patient assessment. As long-acting bronchodilator monotherapy is not recommended for the treatment of patients with asthma, ${ }^{121}$ it is crucial that clinicians are comfortable distinguishing between COPD and asthma. ${ }^{2,3}$ It is tempting to speculate that the overuse of ICS in COPD may be in part driven by the challenge that physicians face in distinguishing between these conditions. ${ }^{77}$

\section{Summary and conclusion}

The 14 fully published, randomized, placebo-controlled studies analyzed here demonstrate that LAMA therapy results in clinically meaningful improvements in lung function and health status, as well as significant reductions in COPD exacerbations and breathlessness when compared with placebo. At present, the greatest weight of evidence exists for tiotropium and GLY, particularly in terms of effects on exacerbations. The few head-to-head studies of GLY and aclidinium vs tiotropium demonstrated generally comparable efficacy in terms of lung function, dyspnea, exacerbations, and health status, with few apparent differences in safety profiles between available LAMAs. Differences exist between the devices used to deliver each LAMA, which may be important to individual patients as well as once- or twice-daily treatment regimen. Therefore, an appropriate choice of initial LAMA therapy should involve individual physician's and patient's experiences and preferences, as well as consideration of patient comorbidities. Furthermore, the availability of a FDC product containing the same agent and using the same device should be considered for the potential future escalation of treatment. Overall, the evidence discussed demonstrates that LAMAs provide effective and generally well-tolerated maintenance therapy for patients with COPD, indicating that these agents can be used with confidence and as first choice in patients typically seen in primary practice.

\section{Acknowledgments}

The authors were assisted in the preparation of the article by Thomas McMurray and Emily Fisher, professional medical writers at CircleScience, an Ashfield Company, part of UDG Healthcare plc. Medical writing support and open access publication charges for this article were funded by Novartis Pharma AG (Basel, Switzerland). We would like to thank Sandy Walmsley for her contributions in the development of this article.

\section{Author contributions}

Authors discussed and agreed to the scope of the article, elaborated the layout of the review and contributed to the development of the article at all stages. All authors contributed toward data analysis, drafting and revising the paper and agree to be accountable for all aspects of the work. All authors read and approved the final article.

\section{Disclosure}

ADD'U has received research, consulting, and lecturing fees from Almirall, Altana, AstraZeneca, Boehringer Inglelheim (Canada) Ltd, Forest Laboratories, GlaxoSmithKline, Merck Canada, KOS Pharmaceuticals, Methapharma, Novartis Canada/USA, ONO Pharma, Pfizer Canada, Schering Plough, Sepracor, and SkyePharma. PK has received personal fees and travel grants from Novartis, AstraZeneca, Bionorica, Boehringer Ingelheim, Chiesi, GlaxoSmithKline, Menarini, Mundipharma, Novartis, and Takeda. RW has received consulting honoraria and travel grants from AstraZeneca, Boehringer Ingelheim, GlaxoSmithKline, Menarini Australia, and Novartis. The authors report no other conflicts of interest in this work.

\section{References}

1. Global Initiative for Chronic Obstructive Lung Disease (GOLD) [webpage on the Internet]. Global Strategy for the Diagnosis, Management, and Prevention of Chronic Obstructive Pulmonary Disease. 2018 Report. 2017. Available from: http://goldcopd.org/gold-reports/. Accessed November 28, 2017.

2. Thomas M, Decramer M, O'Donnell DE. No room to breathe: the importance of lung hyperinflation in COPD. Prim Care Respir J. 2013; 22(1):101-111.

3. Wedzicha JA, Decramer M, Seemungal TA. The role of bronchodilator treatment in the prevention of exacerbations of COPD. Eur Respir J. 2012;40(6):1545-1554.

4. Kew KM, Dias S, Cates CJ. Long-acting inhaled therapy (beta-agonists, anticholinergics and steroids) for COPD: a network meta-analysis. Cochrane Database Syst Rev. 2014;3:CD010844.

5. Buhl R, Dunn LJ, Disdier C, et al. Blinded 12-week comparison of oncedaily indacaterol and tiotropium in COPD. Eur Respir J. 2011;38(4): 797-803. 
6. Vogelmeier C, Hederer B, Glaab T, et al. Tiotropium versus salmeterol for the prevention of exacerbations of COPD. N Engl J Med. 2011; 364(12):1093-1103.

7. Decramer ML, Chapman KR, Dahl R, et al. Once-daily indacaterol versus tiotropium for patients with severe chronic obstructive pulmonary disease (INVIGORATE): a randomised, blinded, parallel-group study. Lancet Respir Med. 2013;1(7):524-533.

8. Electronic Medicines Compendium (eMC) [webpage on the Internet]. Seretide 100, 250, 500 Accuhaler. Summary of Product Characteristics (SmPC); 2015 [updated 2015]. Available from: https://www.medicines. org.uk/emc/medicine/2317. Accessed November 17, 2015.

9. Electronic Medicines Compendium (EMC) [webpage on the Internet]. Eklira Genuair 322 Micrograms Inhalation Powder. Summary of Product Characteristics (SmPC); 2015 [updated 21 May 2015]. Available from: https://www.medicines.org.uk/emc/medicine/27001. Accessed June 15, 2015

10. European Medicines Agency (EMA). Seebri Breezhaler. Summary of Product Characteristics (SmPC); 2015 [updated October 2015]. Available from: http://www.ema.europa.eu/docs/en_GB/document_library/ EPAR_-_Product_Information/human/002430/WC500133769.pdf Accessed October 14, 2015.

11. Electronic Medicines Compendium (EMC) [webpage on the Internet]. Incruse 55 Micrograms inhalation Powder, Pre-dispensed. Summary of Product Characteristics (SmPC); 2015 [updated September 2015] Available from: https://www.medicines.org.uk/emc/medicine/29394. Accessed October 8, 2015.

12. Price D, West D, Brusselle G, et al. Management of COPD in the UK primary-care setting: an analysis of real-life prescribing patterns. Int J Chron Obstruct Pulmon Dis. 2014;9:889-905.

13. Frith PA, Thompson PJ, Ratnavadivel R, et al. Glycopyrronium oncedaily significantly improves lung function and health status when combined with salmeterol/fluticasone in patients with COPD: the GLISTEN study-a randomised controlled trial. Thorax. 2015;70(6):519-527.

14. Singh D, Papi A, Corradi M, et al. Single inhaler triple therapy versus inhaled corticosteroid plus long-acting beta2-agonist therapy for chronic obstructive pulmonary disease (TRILOGY): a double-blind, parallel group, randomised controlled trial. Lancet. 2016;388(10048):963-973.

15. Miravitlles M, Soler-Cataluna JJ, Calle M, et al. Spanish guidelines for management of chronic obstructive pulmonary disease (GesEPOC) 2017. Pharmacological treatment of stable phase. Arch Bronconeumol. 2017;53(6):324-335.

16. European Medicines Agency (EMA). Committee for Medicinal Products for Human Use (CHMP) Assessment Report. Eklira Genuair (aclidinium bromide). Procedure No. EMEA/H/C/002211; 2012. Available from: http://www.ema.europa.eu/docs/en_GB/document_library/ EPAR_-_Public_assessment_report/human/002211/WC500132663. pdf. Accessed June 15, 2015.

17. European Medicines Agency (EMA). Committee for Medicinal Products for Human Use (CHMP) Assessment Report. Incruse (umeclidinium bromide). Procedure No. EMEA/H/C/002809/0000; 2014. Available from: http://www.ema.europa.eu/docs/en_GB/document_library/ EPAR_-_Public_assessment_report/human/002809/WC500167431. pdf. Accessed June 15, 2015.

18. Casaburi R, Mahler DA, Jones PW, et al. A long-term evaluation of once-daily inhaled tiotropium in chronic obstructive pulmonary disease. Eur Respir J. 2002;19(2):217-224.

19. Donohue JF, van Noord JA, Bateman ED, et al. A 6-month, placebocontrolled study comparing lung function and health status changes in COPD patients treated with tiotropium or salmeterol. Chest. 2002; 122(1):47-55.

20. Niewoehner DE, Rice K, Cote C, et al. Prevention of exacerbations of chronic obstructive pulmonary disease with tiotropium, a once-daily inhaled anticholinergic bronchodilator: a randomized trial. Ann Intern Med. 2005;143(5):317-326.

21. Chan CK, Maltais F, Sigouin C, Haddon JM, Ford GT; SAFE Study Group. A randomized controlled trial to assess the efficacy of tiotropium in Canadian patients with chronic obstructive pulmonary disease. Can Respir J. 2007;14(8):465-472.
22. Tashkin DP, Celli B, Senn S, et al. A 4-year trial of tiotropium in chronic obstructive pulmonary disease. N Engl J Med. 2008;359(15):1543-1554.

23. Troosters T, Sciurba FC, Decramer M, et al. Tiotropium in patients with moderate COPD naive to maintenance therapy: a randomised placebo-controlled trial. NPJ Prim Care Respir Med. 2014;24:14003.

24. Bateman E, Singh D, Smith D, et al. Efficacy and safety of tiotropium Respimat SMI in COPD in two 1-year randomized studies. Int J Chron Obstruct Pulmon Dis. 2010;5:197-208.

25. Bateman ED, Tashkin D, Siafakas N, et al. A one-year trial of tiotropium Respimat plus usual therapy in COPD patients. Respir Med. 2010; 104(10):1460-1472.

26. D'Urzo A, Ferguson GT, van Noord JA, et al. Efficacy and safety of once-daily NVA237 in patients with moderate-to-severe COPD: the GLOW1 trial. Respir Res. 2011;12(1):156.

27. Kerwin E, Hébert J, Gallagher N, et al. Efficacy and safety of NVA237 versus placebo and tiotropium in patients with COPD: the GLOW2 study. Eur Respir J. 2012;40(5):1106-1114.

28. Donohue JF, Maleki-Yazdi MR, Kilbride S, Mehta R, Kalberg C, Church A. Efficacy and safety of once-daily umeclidinium/vilanterol 62.5/25 mg in COPD. Respir Med. 2013;107(10):1538-1546.

29. Jones PW, Singh D, Bateman ED, et al. Efficacy and safety of twicedaily aclidinium bromide in COPD patients: the ATTAIN study. Eur Respir J. 2012;40(4):830-836.

30. Singh D, Jones PW, Bateman ED, et al. Efficacy and safety of aclidinium bromide/formoterol fumarate fixed-dose combinations compared with individual components and placebo in patients with COPD (ACLIFORM-COPD): a multicentre, randomised study. BMC Pulm Med. 2014;14(1):178.

31. D'Urzo AD, Rennard SI, Kerwin EM, Mergel V, Leslbaum AR, Caracta CF. Efficacy and safety of fixed-dose combinations of aclidinium bromide/formoterol fumarate: the 24-week, randomized, placebocontrolled AUGMENT COPD study. Respir Res. 2014;15:123.

32. D’Urzo A, Kerwin E, Rennard S, He T, Gil EG, Caracta C. One-year extension study of ACCORD COPD I: safety and efficacy of two doses of twice-daily aclidinium bromide in patients with COPD. COPD. 2013; 10(4):500-510.

33. LaForce C, Pearle J, Feldman G, et al. Efficacy and safety of glycopyrronium in COPD patients with moderate-to-severe airflow limitation: the GEM1 study. Am J Respir Crit Care Med. 2015;257(Meeting Abstracts):A5745.

34. Kerwin E, Siler TM, Korenblat PE, et al. Glycopyrronium twice daily improves lung function and health status and is well tolerated in COPD patients with moderate-to-severe airflow limitation: the GEM2 study. Am J Respir Crit Care Med. 2015;257(Meeting Abstracts):A5742.

35. Menezes AM, Perez-Padilla R, Wehrmeister FC, et al. FEV1 is a better predictor of mortality than FVC: the PLATINO cohort study. PLoS One. 2014;9(10):e109732.

36. Ke X, Marvel J, Yu TC, et al. Impact of lung function on exacerbations, health care utilization, and costs among patients with COPD. Int J Chron Obstruct Pulmon Dis. 2016;11:1689-1703.

37. Donohue JF. Minimal clinically important differences in COPD lung function. COPD. 2005;2(1):111-124.

38. Global Initiative for Chronic Obstructive Lung Disease (GOLD). Spirometry for Healthcare Providers; 2010. Available from: http:// goldcopd.org/wp-content/uploads/2016/04/GOLD_Spirometry_2010. pdf. Accessed December 11, 2017.

39. Celli B, Zuwallack R, Wang S, Kesten S. Improvement in resting inspiratory capacity and hyperinflation with tiotropium in COPD patients with increased static lung volumes. Chest. 2003;124(5):1743-1748.

40. Kostikas K, Siafakas NM. Does the term "deflators" reflect more accurately the beneficial effects of long-acting bronchodilators in COPD? COPD. 2016;13(5):537-539.

41. O'Donnell DE, Fluge T, Gerken F, et al. Effects of tiotropium on lung hyperinflation, dyspnoea and exercise tolerance in COPD. Eur Respir J. 2004;23(6):832-840.

42. Beeh KM, Singh D, Di Scala L, Drollmann A. Once-daily NVA237 improves exercise tolerance from the first dose in patients with COPD: the GLOW3 trial. Int J Chron Obstruct Pulmon Dis. 2012;7:503-513. 
43. Maltais F, Celli B, Casaburi R, et al. Aclidinium bromide improves exercise endurance and lung hyperinflation in patients with moderate to severe COPD. Respir Med. 2011;105(4):580-587.

44. Jones P, Higenbottam T. Quantifying of severity of exacerbations in chronic obstructive pulmonary disease: adaptations to the definition to allow quantification. Proc Am Thorac Soc. 2007;4(8):597-601.

45. Chapman K, Vogelmeier C, FowlerTaylor A, et al. Effect of indacaterol/ glycopyrronium (IND/GLY) vs salmeterol/fluticasone (SFC) on moderate or severe COPD exacerbations and lung function based on baseline blood eosinophil counts: results from the FLAME study. Eur Respir J. 2016;48(suppl 60):PA296.

46. European Respiratory Society (ERS) [webpage on the Internet]. Chapter 13: chronic obstructive pulmonary disease. European Lung White Book; 2016 [updated 2016]. Available from: http://www. erswhitebook.org/chapters/chronic-obstructive-pulmonary-disease/. Accessed November 24, 2016.

47. Jones PW, Singh D, Kerwin E, Lamarca R, Caracta C, Garcia Gil E. Reduced COPD exacerbations associated with aclidinium bromide versus placebo: a pooled analysis of Phase III data. Thorax. 2012;67(suppl 2): A146(Abstract P189).

48. Mahler DA, Wells CK. Evaluation of clinical methods for rating dyspnea. Chest. 1988;93(3):580-586.

49. Mahler DA, Weinberg DH, Wells CK, Feinstein AR. The measurement of dyspnea. Contents, interobserver agreement, and physiologic correlates of two new clinical indexes. Chest. 1984;85(6):751-758.

50. Mahler DA, Witek TJ Jr. The MCID of the transition dyspnea index is a total score of one unit. COPD. 2005;2(1):99-103.

51. Jones PW, Quirk FH, Baveystock CM, Littlejohns P. A self-complete measure of health status for chronic airflow limitation. The St. George's Respiratory Questionnaire. Am Rev Respir Dis. 1992;145(6):1321-1327.

52. Jones PW. St. George's respiratory questionnaire: MCID. COPD. 2005; 2(1):75-79.

53. Jones PW, Beeh KM, Chapman KR, Decramer M, Mahler DA, Wedzicha JA. Minimal clinically important differences in pharmacological trials. Am J Respir Crit Care Med. 2014;189(3):250-255.

54. Chapman KR, Beeh KM, Beier J, et al. A blinded evaluation of the efficacy and safety of glycopyrronium, a once-daily long-acting muscarinic antagonist, versus tiotropium, in patients with COPD: the GLOW5 study. BMC Pulm Med. 2014;14(1):4.

55. Cazzola M, Beeh KM, Price D, Roche N. Assessing the clinical value of fast onset and sustained duration of action of long-acting bronchodilators for COPD. Pulm Pharmacol Ther. 2015;31:68-78.

56. Ismaila AS, Huisman EL, Punekar YS, Karabis A. Comparative efficacy of long-acting muscarinic antagonist monotherapies in COPD: a systematic review and network meta-analysis. Int J Chron Obstruct Pulmon Dis. 2015;10:2495-2517.

57. Karabis A, Lindner L, Mocarski M, Huisman E, Greening A. Comparative efficacy of aclidinium versus glycopyrronium and tiotropium, as maintenance treatment of moderate to severe COPD patients: a systematic review and network meta-analysis. Int J Chron Obstruct Pulmon Dis. 2013;8:405-423.

58. Wedzicha JA, Decramer M, Ficker JH, et al. Analysis of chronic obstructive pulmonary disease exacerbations with the dual bronchodilator QVA149 compared with glycopyrronium and tiotropium (SPARK): a randomised, double-blind, parallel-group study. Lancet Respir Med. 2013;1(3):199-209.

59. Wedzicha JA, Decramer M, Ficker JH, et al. Once-daily glycopyrronium via Breezhaler ${ }^{\circledR}$ device improves lung function and reduces exacerbations in severe-to-very severe COPD patients: the SPARK study. 7th International Primary Care Respiratory Group World Conference. Athens, Greece: 2014. (Abstract OR-081).

60. Wedzicha JA, Agusti A, Donaldson G, Chuecos F, Lamarca R, Garcia Gil E. Effect of aclidinium bromide on exacerbations in patients with moderate-to-severe COPD: a pooled analysis of five phase III, randomized, placebo-controlled studies. COPD. 2016;13(6):669-676.

61. Jones PW, Brusselle G, Dal Negro RW, et al. Health-related quality of life in patients by COPD severity within primary care in Europe. Respir Med. 2011;105(1):57-66.
62. Jones RC, Price D, Ryan D, et al. Opportunities to diagnose chronic obstructive pulmonary disease in routine care in the UK: a retrospective study of a clinical cohort. Lancet Respir Med. 2014;2(4):267-276.

63. Kardos P, Vogelmeier C, Buhl R, Criee CP, Worth H. The Prospective Non-Interventional DACCORD Study in the National COPD Registry in Germany: design and methods. BMC Pulm Med. 2015;15:2.

64. Worth H, Buhl R, Criee CP, Kardos P, Mailander C, Vogelmeier C. The 'real-life' COPD patient in Germany: the DACCORD study. Respir Med. 2016;111:64-71.

65. Miravitlles M, Price D, Rabe KF, Schmidt H, Metzdorf N, Celli B. Comorbidities of patients in tiotropium clinical trials: comparison with observational studies of patients with chronic obstructive pulmonary disease. Int J Chron Obstruct Pulmon Dis. 2015;10:549-564.

66. Vanfleteren LE, Spruit MA, Groenen M, et al. Clusters of comorbidities based on validated objective measurements and systemic inflammation in patients with chronic obstructive pulmonary disease. Am J Respir Crit Care Med. 2013;187(7):728-735.

67. Travers J, Marsh S, Caldwell B, et al. External validity of randomized controlled trials in COPD. Respir Med. 2007;101(6):1313-1320.

68. Redelmeier DA, Tan SH, Booth GL. The treatment of unrelated disorders in patients with chronic medical diseases. N Engl J Med. 1998; 338(21):1516-1520.

69. Polkey MI, Rabe KF. Chicken or egg: physical activity in COPD revisited. Eur Respir J. 2009;33(2):227-229.

70. Seemungal TA, Donaldson GC, Paul EA, Bestall JC, Jeffries DJ, Wedzicha JA. Effect of exacerbation on quality of life in patients with chronic obstructive pulmonary disease. Am J Respir Crit Care Med. 1998;157(5 pt 1):1418-1422.

71. Pitta F, Troosters T, Probst VS, Spruit MA, Decramer M, Gosselink R. Physical activity and hospitalization for exacerbation of COPD. Chest. 2006;129(3):536-544.

72. Donaldson GC, Seemungal TA, Bhowmik A, Wedzicha JA. Relationship between exacerbation frequency and lung function decline in chronic obstructive pulmonary disease. Thorax. 2002;57(10):847-852.

73. Kanner RE, Anthonisen NR, Connett JE. Lower respiratory illnesses promote $\mathrm{FEV}_{1}$ decline in current smokers but not ex-smokers with mild chronic obstructive pulmonary disease: results from the lung health study. Am J Respir Crit Care Med. 2001;164(3):358-364.

74. Soler-Cataluña JJ, Martínez-García MA, Román Sánchez P, Salcedo E, Navarro M, Ochando R. Severe acute exacerbations and mortality in patients with chronic obstructive pulmonary disease. Thorax. 2005; 60(11):925-931.

75. Connors AF Jr, Dawson NV, Thomas C, et al. Outcomes following acute exacerbation of severe chronic obstructive lung disease. The SUPPORT investigators (Study to Understand Prognoses and Preferences for Outcomes and Risks of Treatments). Am J Respir Crit Care Med. 1996; 154(4 pt 1):959-967.

76. Rabe KF, Fabbri LM, Israel E, et al. Effect of ADRB2 polymorphisms on the efficacy of salmeterol and tiotropium in preventing COPD exacerbations: a prespecified substudy of the POET-COPD trial. Lancet Respir Med. 2014;2(1):44-53.

77. D’Urzo A, Donohue JF, Kardos P, Miravitlles M, Price D. A reevaluation of the role of inhaled corticosteroids in the management of patients with chronic obstructive pulmonary disease. Expert Opin Pharmacother. 2015;16(12):1845-1860.

78. D’Urzo A, Kerwin E, Overend T, D'Andrea P, Chen H, Goyal P. Once daily glycopyrronium for the treatment of COPD: pooled analysis of the GLOW1 and GLOW2 studies. Curr Med Res Opin. 2014;30(3): 493-508.

79. Bateman ED, Ferguson GT, Barnes N, et al. Dual bronchodilation with QVA149 versus single bronchodilator therapy: the SHINE study. Eur Respir J. 2013;42(6):1484-1494.

80. Beeh KM, Westerman J, Kirsten AM, et al. The 24-h lung-function profile of once-daily tiotropium and olodaterol fixed-dose combination in chronic obstructive pulmonary disease. Pulm Pharmacol Ther. 2015;32:53-59.

81. van Noord JA, Aumann JL, Janssens E, et al. Comparison of tiotropium once daily, formoterol twice daily and both combined once daily in patients with COPD. Eur Respir J. 2005;26(2):214-222. 
82. van Noord JA, Aumann JL, Janssens E, et al. Effects of tiotropium with and without formoterol on airflow obstruction and resting hyperinflation in patients with COPD. Chest. 2006;129(3):509-517.

83. Mahler DA, Keininger DL, Mezzi K, Fogel R, Banerji D. Efficacy of indacaterol/glycopyrronium in patients with COPD who have increased dyspnea with daily activities. Chronic Obstr Pulm Dis. 2016; 3(4):758-768.

84. Bateman ED, Mahler DA, Vogelmeier CF, Wedzicha JA, Patalano F, Banerji D. Recent advances in COPD disease management with fixed-dose long-acting combination therapies. Expert Rev Respir Med. 2014;8(3):357-379.

85. Wedzicha JA, Calverley PM, Seemungal TA, et al. The prevention of chronic obstructive pulmonary disease exacerbations by salmeterol/ fluticasone propionate or tiotropium bromide. Am J Respir Crit Care Med. 2008;177(1):19-26.

86. Wedzicha JA, Banerji D, Chapman KR, et al. Indacaterol-glycopyrronium versus salmeterol-fluticasone for COPD. $N$ Engl J Med. 2016;374(23):2222-2234.

87. Lipson DA, Barnacle H, Birk R, et al. FULFIL trial: once-daily triple therapy in patients with chronic obstructive pulmonary disease. Am J Respir Crit Care Med. 2017;196(4):438-446.

88. Pascoe SJ, Lipson DA, Locantore N, et al. A phase III randomised controlled trial of single-dose triple therapy in COPD: the IMPACT protocol. Eur Respir J. 2016;48(2):320-330.

89. Magnussen H, Tetzlaff K, Bateman ED, et al. Lung function changes over time following withdrawal of inhaled corticosteroids in patients with severe COPD. Eur Respir J. 2016;47(2):651-654

90. Calverley PM. COPD therapy: if two is good, is three better? Lancet. 2016;388(10048):937-938.

91. Martinez FJ, Boscia J, Feldman G, et al. Fluticasone furoate/vilanterol (100/25; 200/25 mg) improves lung function in COPD: a randomised trial. Respir Med. 2013;107(4):550-559.

92. Kerwin EM, Scott-Wilson C, Sanford L, et al. A randomised trial of fluticasone furoate/vilanterol (50/25 mg; 100/25 mg) on lung function in COPD. Respir Med. 2013;107(4):560-569.

93. Magnussen H, Disse B, Rodriguez-Roisin R, et al. Withdrawal of inhaled glucocorticoids and exacerbations of COPD. $N$ Engl J Med. 2014;371(14):1285-1294.

94. Siafakas N, Corlateanu A, Fouka E. Phenotyping before starting treatment in COPD? COPD. 2017;14(3):367-374.

95. Pascoe S, Locantore N, Dransfield MT, Barnes NC, Pavord ID. Blood eosinophil counts, exacerbations, and response to the addition of inhaled fluticasone furoate to vilanterol in patients with chronic obstructive pulmonary disease: a secondary analysis of data from two parallel randomised controlled trials. Lancet Respir Med. 2015;3(6): 435-442.

96. Pavord ID, Lettis S, Locantore N, et al. Blood eosinophils and inhaled corticosteroid/long-acting beta-2 agonist efficacy in COPD. Thorax. 2016;71(2):118-125.

97. Siddiqui SH, Guasconi A, Vestbo J, et al. Blood eosinophils: a biomarker of response to extrafine beclomethasone/formoterol in chronic obstructive pulmonary disease. Am J Respir Crit Care Med. 2015; 192(4):523-525.

98. Roche N, Chapman KR, Vogelmeier CF, et al. Blood eosinophils and response to maintenance COPD treatment: data from the FLAME trial. Am J Respir Crit Care Med. 2017;195(9):1189-1197.

99. Miravitlles M, Anzueto A. A new two-step algorithm for the treatment of COPD. Eur Respir J. 2017;49(2).

100. Miravitlles M, D’Urzo A, Singh D, Koblizek V. Pharmacological strategies to reduce exacerbation risk in COPD: a narrative review. Respir Res. 2016;17(1):112.

101. Colthorpe P, Voshaar T, Kiekbusch T, Cuoghi E, Jauernig J. Delivery characteristics of a low-resistance dry-powder inhaler used to deliver the long-acting muscarinic antagonist glycopyrronium. J Drug Assess 2013;2(1):11-16.

102. Lyseng-Williamson KA, Keating GM. Tiotropium Respimat ${ }^{\circledR}$ Soft Mist ${ }^{\mathrm{TM}}$ inhaler: a guide to its use in chronic obstructive pulmonary disease (COPD) in the EU. Drugs Ther Perspect. 2015;31:39-44.
103. Gjaltema D, Hagedoorn P, Grasmeijer F, Huijbers BG, Frijlink HW de Boer AH. Comparative in vitro performance of the new drug aclidinium in a novel multidose dry powder inhaler. Eur Respir J. 2013; 42(suppl 57):692s(Abstract P3384).

104. Svedsater H, Dale P, Garrill K, Walker R, Woepse MW. Qualitative assessment of attributes and ease of use of the ELLIPTA ${ }^{\mathrm{TM}}$ dry powder inhaler for delivery of maintenance therapy for asthma and COPD BMC Pulm Med. 2013;13:72.

105. Grant AC, Walker R, Hamilton M, Garrill K. The ELLIPTA $®$ dry powder inhaler: design, functionality, in vitro dosing performance and critical task compliance by patients and caregivers. J Aerosol Med Pulm Drug Deliv. 2015;28(6):474-485.

106. Chrystyn H, Niederlaender C. The Genuair ${ }^{\circledR}$ inhaler: a novel, multidose dry powder inhaler. Int J Clin Pract. 2012;66(3):309-317.

107. Molimard M, Raherison C, Lignot-Maleyran S, et al. Chronic obstructive pulmonary disease exacerbation and inhaler device handling: real-life assessment of 2935 patients. Eur Respir J. 2017;49: 1601794

108. Ingebrigtsen TS, Marott JL, Nordestgaard BG, et al. Low use and adherence to maintenance medication in chronic obstructive pulmonary disease in the general population. J Gen Intern Med. 2015;30(1): 51-59.

109. Mehuys E, Boussery K, Adriaens E, et al. COPD management in primary care: an observational, community pharmacy-based study. Ann Pharmacother. 2010;44(2):257-266.

110. Mäkelä MJ, Backer V, Hedegaard M, Larsson K. Adherence to inhaled therapies, health outcomes and costs in patients with asthma and COPD. Respir Med. 2013;107(10):1481-1490.

111. Bereza BG, Troelsgaard Nielsen A, Valgardsson S, Hemels ME, Einarson TR. Patient preferences in severe COPD and asthma: a comprehensive literature review. Int J Chron Obstruct Pulmon Dis. 2015;10: 739-744.

112. Molimard M, Colthorpe P. Inhaler devices for chronic obstructive pulmonary disease: insights from patients and healthcare practitioners. J Aerosol Med Pulm Drug Deliv. 2015;28(3):219-228.

113. Price D, Lee AJ, Sims EJ, et al. Characteristics of patients preferring once-daily controller therapy for asthma and COPD: a retrospective cohort study. Prim Care Respir J. 2013;22(2):161-168.

114. Bourbeau J, Bartlett SJ. Patient adherence in COPD. Thorax. 2008; 63(9):831-838.

115. Dederichs J, Singh D, Pavkov R. Inspiratory flow profiles generated by patients with COPD through the Breezhaler ${ }^{\circledR}$ inhaler and other marketed dry powder inhalers. Am J Respir Crit Care Med. 2015;191: A5793

116. Chapman KR, Fogarty CM, Peckitt C, et al. Delivery characteristics and patients' handling of two single-dose dry-powder inhalers used in COPD. Int J Chron Obstruct Pulmon Dis. 2011;6:353-363.

117. Komase Y, Asako A, Kobayashi A, Sharma R. Ease-of-use preference for the ELLIPTA(R) dry powder inhaler over a commonly used singledose capsule dry powder inhaler by inhalation device-naive Japanese volunteers aged 40 years or older. Int J Chron Obstruct Pulmon Dis. 2014;9:1365-1375

118. Pascual S, Feimer J, De Soyza A, et al. Preference, satisfaction and critical errors with Genuair and Breezhaler inhalers in patients with COPD: a randomised, cross-over, multicentre study. NPJ Prim Care Respir Med. 2015;25:15018.

119. van der Palen J, Ginko T, Kroker A, et al. Preference, satisfaction and errors with two dry powder inhalers in patients with COPD. Expert Opin Drug Deliv. 2013;10(8):1023-1031.

120. Hanania NA, Celli BR, Donohue JF, Martin UJ. Bronchodilator reversibility in COPD. Chest. 2011;140(4):1055-1063.

121. Global Initiative for Asthma (GINA) [webpage on the Internet]. 2017 Pocket Guide for Asthma Management and Prevention; 2017. Available from: http://ginasthma.org/2017-pocket-guide-for-asthmamanagement-and-prevention. Accessed December 11, 2017. 
International Journal of COPD

Dovepress

\section{Publish your work in this journal}

The International Journal of COPD is an international, peer-reviewed journal of therapeutics and pharmacology focusing on concise rapid reporting of clinical studies and reviews in COPD. Special focus is given to the pathophysiological processes underlying the disease, intervention programs, patient focused education, and self management protocols.

This journal is indexed on PubMed Central, MedLine and CAS. The manuscript management system is completely online and includes a very quick and fair peer-review system, which is all easy to use. Visit http://www.dovepress.com/testimonials.php to read real quotes from published authors.

Submit your manuscript here: http://www.dovepress.com/international-journal-of-chronic-obstructive-pulmonary-disease-journal 